\title{
Hunting Stability of High-Speed Railway Vehicles Under Steady Aerodynamic Loads
}

\author{
Xiao-Hui Zeng* \\ Key Laboratory for Mechanics in Fluid Solid Coupling Systems \\ Institute of Mechanics, Chinese Academy of Sciences \\ Beijing 100190, P. R. China \\ School of Engineering Science \\ University of Chinese Academy of Sciences \\ Beijing 100049, P. R. China \\ zxh@imech.ac.cn \\ Jiang Lai \\ Nuclear Power Institute of China \\ Chengdu 610041, P. R. China \\ Han Wu \\ Key Laboratory for Mechanics in Fluid Solid Coupling Systems \\ Institute of Mechanics, Chinese Academy of Sciences \\ Beijing 100190, P. R. China \\ School of Engineering Science \\ University of Chinese Academy of Sciences \\ Beijing 100049, P. R. China
}

Received 1 May 2017

Accepted 20 November 2017

Published 18 December 2017

\begin{abstract}
With the rising speed of high-speed trains, the aerodynamic loads become more significant and their influences on the hunting stability of railway vehicles deserve to be considered. Such an effect cannot be properly considered by the conventional model of hunting stability analysis. To this end, the linear hunting stability of high-speed railway vehicles running on tangent tracks is studied. A model considering the steady aerodynamic loads due to the joint action of the airflow facing the moving train and the crosswind, is proposed for the hunting stability analysis of a railway vehicle with 17 degrees of freedom (DOF). The key factors considered include: variations of the wheel-rail normal forces, creep coefficients, gravitational stiffness and angular stiffness due to the actions of the aerodynamic load, which affects the characteristics of hunting stability. Using the computer program developed, numerical calculations were carried out for studying the behavior of the linear hunting stability of vehicles under steady aerodynamic loads.
\end{abstract}

\footnotetext{
* Corresponding author.
} 


\begin{abstract}
The results show that the aerodynamic loads have an obvious effect on the linear critical speeds and instability modes. The linear critical speed decreases monotonously as the crosswind velocity increases, and the influences of pitch moment and lift force on the linear critical speed are larger than the other components of the aerodynamic loads.
\end{abstract}

Keywords: Stability; self-excited vibration; high-speed railway; steady aerodynamic load; hunting; multibody dynamics.

\title{
1. Introduction
}

High-speed passenger trains are being increasingly operated to cope with the everincreasing hunger for bigger transport capacity. A speed record of $574.8 \mathrm{~km} / \mathrm{h}$ for wheeled trains was set on April 3, 2007 by a French TGV test train on conventional rails. Among the wheeled trains in service, a Chinese CRH380BL set reached $487.3 \mathrm{~km} / \mathrm{h}$ on the Beijing-Shanghai high-speed railway during the trial service on January 10, 2011. Besides the notable increase in the uppermost speed, the sustainable operation speed is also boosted. The maximum speed of the China railway high-speed $(\mathrm{CRH})$ trains in the Beijing-Tianjin intercity rail line, the WuhanGuangzhou and Zhengzhou-Xi'an passenger dedicated lines, and the ShanghaiNanjing high-speed railway, is $380 \mathrm{~km} / \mathrm{h}$ during regular commercial operations, whereas the operating speed is $350 \mathrm{~km} / \mathrm{h}$.

Speed boosts may cause problems that differ from those at low speed. One of the problems has to do with the effects of the aerodynamic load on the train. There have been many studies on this subject. Suzuki et al. ${ }^{1}$ evaluated the aerodynamic characteristics of the typical vehicle configurations on embankment by using tunnel tests. Carrarini $^{2}$ studied the effects of aerodynamic loads on vehicles by modeling the parameters as stochastic variables. Baker ${ }^{3}$ investigated the crosswind effect on train and presented a simple method for calculating the wind time history. Bocciolone et $a .^{4}{ }^{4}$ carried out extensive wind-tunnel tests on three types of railway vehicles and performed sensitivity analysis on the parameters influencing the aerodynamic behavior. Baker and $\mathrm{Cheli}^{5}$ reviewed the research findings on the crosswind aerodynamic effects on road and railway vehicles and set out the methodology for predicting accident risks by using aerodynamic force characteristics. Wetzel and Proppe ${ }^{6}$ performed the probabilistic analysis of the railway vehicle systems considering the turbulent crosswind excitation. Thomas et $a l .^{7}$ carried out multibody simulations for a high-speed railway vehicle in crosswind and studied the effects of aerodynamic loads induced by crosswind on a vehicle negotiating a curve. Cheng et al. ${ }^{8}$ investigated the dynamic responses of a railway vehicle subjected to three-directional wind-induced forces and moments. Baker et al. ${ }^{9}$ presented a method to produce aerodynamic force time histories by using wind-tunnel testing and computational fluid dynamics, followed by vehicle dynamics simulations. Cheli et al. ${ }^{10}$ and Tomasini and Cheli ${ }^{11}$ presented a numerical model for the aerodynamic admittance function, and verified the model based on experimental data. 
The studies regarding the effects of aerodynamic loads on trains may be broadly divided into two categories. The first is concerned with seeking the characteristics of the flow field and the aerodynamic force or moment coefficients by using experiments or computational fluid dynamics, neglecting the wheel-rail interaction. The second concerns the vehicle behavior, especially derailment risk indicators, under aerodynamic load. On this subject, besides the majority of studies on the characteristics of flow fields and aerodynamic issues, nearly all other works on dynamic responses of the vehicle are concerned with the forced vibration induced by aerodynamic loads. Little has been done on the effect of aerodynamic load on the self-excited vibration or "hunting phenomenon" of railway vehicles.

The hunting phenomenon is an important feature of the motion of railway vehicles that has to be tested in any newly designed vehicle before service. The critical speed at which railroad vehicles experience severe hunting is critical in safety design, because of the potential instability and the associated passenger discomfort or possibly derailment.

Wickens ${ }^{12}$ investigated the hunting stability of wheelsets and bogies, and analyzed the effects of various parameters. Hannebrink et al. ${ }^{13}$ studied the effects of axle load, track gage, and wheel profile on the wheelset hunting. Tuten et al. ${ }^{14}$ analyzed the effects of different wheel profiles and asymmetric loading on the lateral stability of freight cars. Hirotsu et al. ${ }^{15}$ studied the hunting stability of a railway vehicle with 17 degrees of freedom (DOF). Knudsen et al. ${ }^{16}$ presented a numerical model for analyzing the stability of a wheelset. Zeng ${ }^{17}$ numerically studied the Hopf bifurcation and limit cycles of a nonlinear railway vehicle system. Zhang and Shen ${ }^{18}$ analyzed the periodic motion stability of a nonlinear vehicle system. True ${ }^{19}$ discussed the nonlinear characteristics of railway vehicle systems based on nonlinear dynamics. Yabuno et $a l .{ }^{20}$ used the center manifold theory and the normal form method to study the nonlinear characteristics of hunting motion of a railway wheelset. Lee and Cheng $^{21,22}$ presented a model of the truck with 10 DOF and showed that the critical hunting speeds differ significantly from those of a six-DOF system. Cheng et al. ${ }^{23}$ examined the hunting stability of a vehicle during curving, and presented the respective effects of the various parameters on the critical speed. Kim and Seok ${ }^{24}$ performed bifurcation analysis on the hunting behavior of a nonlinear railway vehicle with dual bogies. Zboinski and Dusza ${ }^{25}$ studied the nonlinear stability of a railway vehicle in a curved track. Zhai and $\mathrm{Wang}^{26}$ studied the lateral hunting stability of railway vehicles considering track elasticity. Polach and Kaiser ${ }^{27}$ used the pathfollowing and brute-force methods to analyze the bifurcation and hunting of a complex railway vehicle. Dong and Zeng ${ }^{28}$ proposed an algebraic approach based on the normal form method to determine the bifurcation mode (subcritical or supercritical) of the lateral stability of the wheelset of a railway vehicle.

As mentioned above, with the significantly increasing train speeds, the aerodynamic load becomes important. Then, the hunting behavior of high-speed railway vehicles may differ from the low-speed equivalent. Despite the number of studies on 
the hunting of railway vehicles, few have considered the influence of aerodynamic loads. Therefore, it is still a problem deserving further investigations for the effects of aerodynamic loads on the hunting stability of high-speed railway vehicles. That is to say, what effects can the high-speed air flow in the opposite advancing direction of the train (in addition to the wind blowing across the direction of travel) have on the hunting stability and the variation rules of suspension parameters versus critical speed? Quantitative study has to be performed on this problem because the aerodynamic loads increase very fast as the speed of the air flow boosts.

In this study, we investigated the eigenvalue problem of the hunting stability of a high-speed railway vehicle traveling on a straight track, considering steady aerodynamic loads (lift, drag, lateral forces, roll moment, and pitch moment) owing to the airflow opposite to the direction of the train and the wind across the direction of travel. The effects of variations of wheel-track normal forces, creep coefficients, and gravitational stiffness and angular stiffness owing to the aerodynamic load were taken into account. A mathematical model incorporating such effects due to aerodynamic loads for the hunting stability analysis of a vehicle with 17 DOF is presented. A computer code based on the presented model was written. Comparison and verification were performed by recalculations of published degenerate examples. By using the code, numerical calculations (incorporating effects of aerodynamic loads) for eigenvalues, eigenvectors, root locus diagram, and linear critical hunting speed in different cases were carried out. We also show the variation trends of critical speed with respect to suspension parameters, under the aerodynamic load in different airflow fields.

In this paper, we only focus on the effect of steady aerodynamic load. The effect of unsteady aerodynamic load will be discussed in a future study.

\section{Mathematical Model for the Hunting Stability of Railway Vehicles Under the Aerodynamic Load}

To analyze the hunting stability of high-speed railway vehicles, we consider the vehicles as a multibody system consisting of wheelsets, bogie frames, and car bodies. The wheelsets and bogie frames are connected by the primary suspensions, and the bogie frames and car bodies are connected by the secondary suspensions, as shown in Fig. 1.

The following notations are used.

$y_{c}, y_{f i}, y_{w i}$ : lateral displacements of car body, bogie frame and wheelset;

$\varphi_{c}, \varphi_{f i}, \varphi_{w i}$ : yaw angles of car body, bogie frame and wheelset;

$\theta_{c}, \theta_{f i}:$ roll angles of car body and bogie frame;

$y_{d i}$ : lateral displacement of spring-damping connecting point of lateral damper;

$x_{d L i}, x_{d R i}$ : longitudinal displacements of the spring-damping connecting point of yaw damper;

$M_{c}, M_{f}, M_{w}$ : masses of car body, bogie frame, and wheelset; 


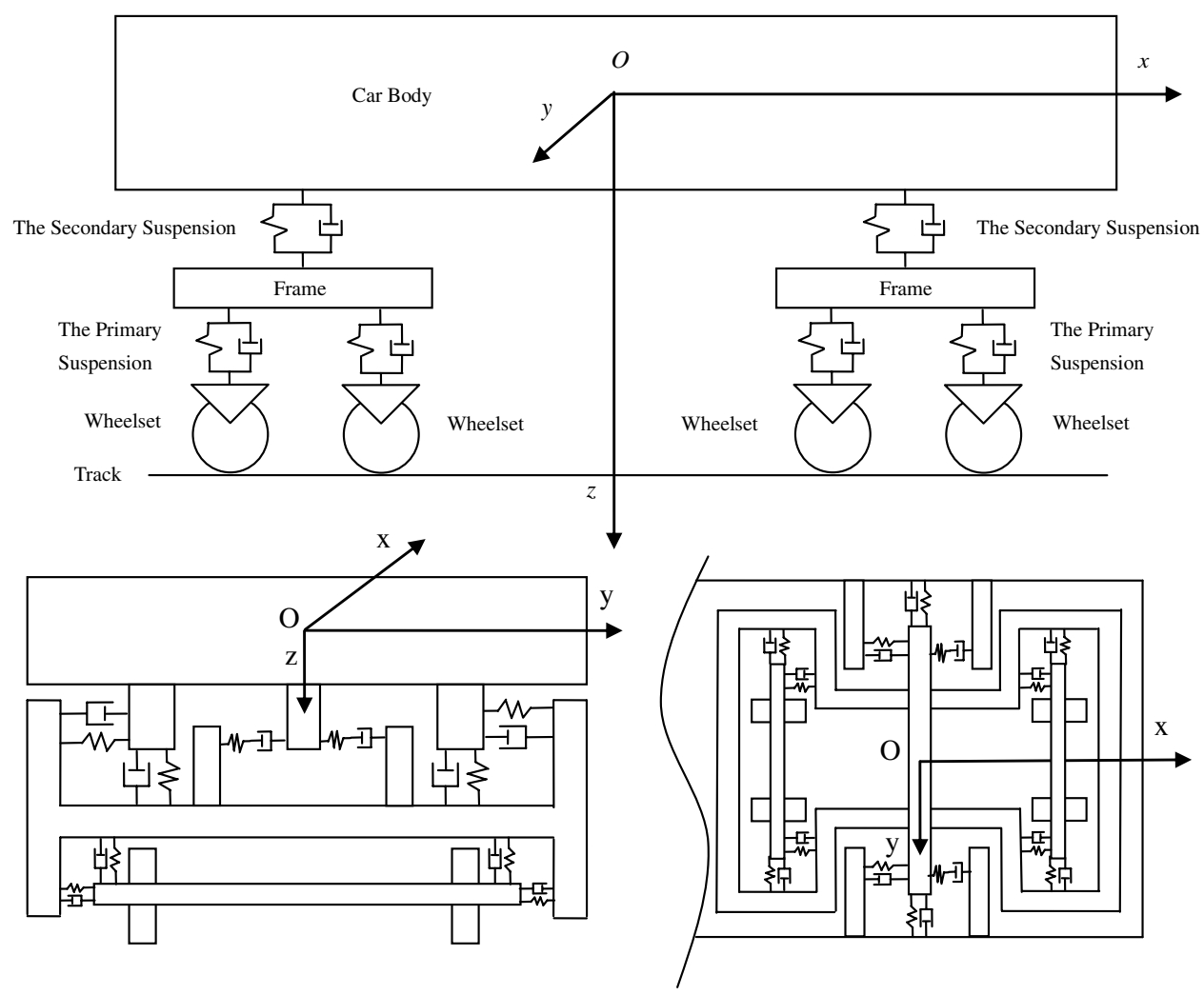

Fig. 1. Dynamic model of a railway vehicle.

$I_{c z}, I_{f z}, I_{w}$ : yaw moments of inertia of car body, bogie frame and wheelset;

$I_{c x}, I_{f x}$ : roll moments of inertia of car body and bogie frame;

$K_{w x}, K_{w y}$ : longitudinal and lateral supporting stiffnesses of the primary suspension;

$K_{1}, C_{1}$ : vertical stiffness and damping of the primary suspension;

$K_{2}, C_{2}$ : vertical stiffness and damping of the secondary suspension;

$K_{2}^{\prime}$ : lateral and longitudinal stiffness of the secondary suspension;

$K_{d}, K_{y}$ : supporting stiffnesses of the yaw and lateral dampers;

$C_{d}, C_{y}$ : dampings of the yaw and lateral dampers;

$h_{f}$ : vertical distance from the center of gravity (C.G.) of the bogie frame to the primary suspension;

$h_{1}$ : vertical distance from the C.G. of the bogie frame to the secondary suspension;

$h_{2}$ : vertical distance from the C.G. of car body to the secondary suspension;

$h_{3}$ : vertical distance from the C.G. of the bogie frame to lateral damper;

$h_{4}$ : vertical distance from the C.G. of the car body to lateral damper;

$a$ : half of wheelbase;

$b$ : half of the distance between tracks;

$b_{1}$ : half of the lateral distance between primary suspensions; 
$b_{2}$ : half of the lateral distance between secondary suspensions;

$b_{3}$ : half of the distance between lateral dampers;

$l$ : half of the distance between bogie centers;

$R$ : rolling radius of wheel;

$\Delta R_{R i}, \Delta R_{L i}$ : rolling radius deviations from the nominal value $R$;

$N_{R i}, N_{L i}$ : wheel-rail normal forces;

$\delta_{R i}, \delta_{L i}:$ wheel-rail contact angles;

$f_{11 R i}, f_{11 L i}, f_{22 R i}, f_{22 L i}$ : longitudinal and lateral creep force coefficients;

$V$ : vehicle speed.

There are $17 \mathrm{DOF}$ in this multibody system including the lateral displacements and yaw angles of the four wheelsets $\left(y_{w}\right.$ and $\left.\varphi_{w}\right)$, the lateral displacements, yaw, and roll of the two bogie frames $\left(y_{f}, \varphi_{f}\right.$ and $\left.\theta_{f}\right)$, the lateral displacements, yaw, and roll of the car body $\left(y_{c}, \varphi_{c}\right.$ and $\left.\theta_{c}\right)$. In addition, we considered the six displacements of the damper rubber joints, including the longitudinal displacements of the springdamping connecting point of four yaw dampers $\left(x_{d}\right)$, and the lateral displacements of the spring-damping connecting point of two lateral dampers $\left(x_{y}\right)$. The differential equations of motion for the hunting of railway vehicles are the following.

For the first and second wheelsets $(i=1,2)$

$$
\begin{aligned}
& M_{w} \ddot{y}_{w i}+2 K_{w y}\left(y_{w i}-y_{f 1} \mp a \varphi_{f 1}-h_{f} \theta_{f 1}\right)+2 f_{22}\left(\frac{\dot{y}_{w i}}{V}-\varphi_{w i}\right)+\frac{W \lambda}{b} y_{w i}=0, \\
& I_{w} \ddot{\varphi}_{w i}+2 K_{w x} b_{1}^{2}\left(\varphi_{w i}-\varphi_{f 1}\right)+2 f_{11}\left(\frac{b \lambda}{R} y_{w i}+\frac{b^{2}}{V} \dot{\varphi}_{w i}\right)-W \lambda b \varphi_{w i}=0 .
\end{aligned}
$$

For the third and fourth wheelsets $(i=3,4)$

$$
\begin{aligned}
& M_{w} \ddot{y}_{w i}+2 K_{w y}\left(y_{w i}-y_{f 2} \mp a \varphi_{t 2}-h_{f} \theta_{f 2}\right)+2 f_{22}\left(\frac{\dot{y}_{w i}}{V}-\varphi_{w i}\right)+\frac{W \lambda}{b} y_{w i}=0, \\
& I_{w} \ddot{\varphi}_{w i}+2 K_{w x} b_{1}^{2}\left(\varphi_{w i}-\varphi_{f 2}\right)+2 f_{11}\left(\frac{b \lambda}{R} y_{w i}+\frac{b^{2}}{V} \dot{\varphi}_{w i}\right)-W \lambda b \varphi_{w i}=0 .
\end{aligned}
$$

In Eqs. (1) and (2), subscript $i=1,2,3$, and 4 denotes the $i$ th wheelset, respectively.

For the bogie frames $(j=1,2)$

$$
\begin{aligned}
& M_{f} \ddot{y}_{f j}-2 K_{w y}\left(y_{w(2 j-1)}+y_{w(2 j)}-2 y_{f j}-2 h_{f} \theta_{f j}\right)-2 K_{2}^{\prime}\left(y_{c} \pm l \varphi_{c}+h_{2} \theta_{c}\right. \\
& \left.\quad-y_{f j}+h_{1} \theta_{f j}\right)+K_{y}\left(y_{d 1}-y_{c} \mp l \varphi_{c}-h_{4} \theta_{c}\right)=0, \\
& \quad I_{f z} \ddot{\varphi}_{f j}-2 a K_{w y}\left(y_{w(2 j-1)}-y_{w(2 j)}-2 a \varphi_{f j}\right)-2 K_{w x} b_{1}^{2}\left(\varphi_{w(2 j-1)}+\varphi_{w(2 j)}-2 \varphi_{f j}\right) \\
& \quad-2 K_{2}^{\prime} b_{2}^{2}\left(\varphi_{c}-\varphi_{f j}\right)+b_{3} K_{d}\left(x_{d R j}-x_{d L j}-2 b_{3} \varphi_{c}\right)=0, \\
& \quad I_{f x} \ddot{\theta}_{f j}-2 h_{f} K_{w y}\left(y_{w(2 j-1)}+y_{w(2 j)}-2 y_{f j}-2 h_{f} \theta_{f j}\right)+4 b_{1}^{2}\left(K_{1} \theta_{f j}+C_{1} \dot{\theta}_{f j}\right) \\
& \quad-2 b_{2}^{2}\left(K_{2}\left(\theta_{c}-\theta_{f j}\right)+C_{2}\left(\dot{\theta}_{c}-\dot{\theta}_{f j}\right)\right)+2 h_{1} K_{2}^{\prime}\left(y_{c} \pm l \varphi_{c}+h_{2} \theta_{c}-y_{f j}+h_{1} \theta_{f j}\right) \\
& \quad+h_{3} K_{y}\left(y_{d j}-y_{c} \mp l \varphi_{c}-h_{4} \theta_{c}\right)=0 .
\end{aligned}
$$


Subscripts $j=1$ and 2 in Eq. (3) denotes the front and rear frames, respectively.

For the body,

$$
\begin{aligned}
M_{c} \ddot{y}_{c} & +2 K_{2}^{\prime}\left(2 y_{c}+2 h_{2} \theta_{c}-y_{f 1}-y_{f 2}+h_{1} \theta_{f 1}+h_{1} \theta_{f 2}\right) \\
& -K_{y}\left(y_{d 1}+y_{d 2}-2 y_{c}-2 h_{4} \theta_{c}\right)=0 \\
I_{c z} \ddot{\varphi}_{c} & +2 l K_{2}^{\prime}\left(2 l \varphi_{c}-y_{f 1}+y_{f 2}+h_{1} \theta_{f 1}-h_{1} \theta_{f 2}\right) \\
& -l K_{y}\left(y_{d 1}-y_{d 2}-2 l \varphi_{c}\right)+2 b_{2}^{2} K_{2}^{\prime}\left(2 \varphi_{c}-\varphi_{f 1}-\varphi_{f 2}\right) \\
& +b_{3} K_{d}\left(x_{d L 1}-x_{d R 1}+x_{d L 2}-x_{d R 2}+4 b_{3} \varphi_{c}\right)=0 \\
\ddot{\theta}_{c x} & +2 b_{2}^{2}\left[K_{2}\left(2 \theta_{c}-\theta_{f 1}-\theta_{f 2}\right)+C_{2}\left(2 \dot{\theta}_{c}-\dot{\theta}_{f 1}-\dot{\theta}_{f 2}\right)\right] \\
& +2 h_{2} K_{2}^{\prime}\left(2 y_{c}+2 h_{2} \theta_{c}-y_{f 1}-y_{f 2}+h_{1} \theta_{f 1}+h_{1} \theta_{f 2}\right) \\
& -h_{4} K_{y}\left(y_{d 1}+y_{d 2}-2 y_{c}-2 h_{4} \theta_{c}\right)=0 .
\end{aligned}
$$

For the spring-damping connecting points of the lateral dampers $(i=1,2)$,

$$
\dot{y}_{d j}=-\frac{K_{y}\left(y_{b 1}-y_{c} \mp l \varphi_{c}-h_{4} \theta_{c}\right)}{C_{y}}+\dot{y}_{f j}+h_{3} \dot{\theta}_{f j} .
$$

For the spring-damping connecting points of the yaw dampers $(i=1,2)$,

$$
\begin{aligned}
& \dot{x}_{d L j}=-\frac{K_{d}\left(x_{d L j}+b_{3} \varphi_{c}\right)}{C_{d}}-b_{3} \dot{\varphi}_{f j}, \\
& \dot{x}_{d R j}=-\frac{K_{d}\left(x_{d R j}-b_{3} \varphi_{c}\right)}{C_{d}}+b_{3} \dot{\varphi}_{f j} .
\end{aligned}
$$

In Eqs. (5) and (6), subscripts $j=1$ and 2 denote the dampers on the front and rear frames, respectively.

In the shorthand of matrix analysis, the 17 DOF of the vehicle are expressed as $\mathbf{Y}_{1}$, the six displacements of the damper rubber joint are expressed as $\mathbf{Y}_{2}$, and the differential equations of motion are given by Eqs. (7)-(9).

$$
\begin{aligned}
& \mathbf{Y}_{1}=\left[\begin{array}{lllllllllllllllll}
y_{w 1} & y_{w 2} & y_{w 3} & y_{w 4} & \varphi_{w 1} & \varphi_{w 2} & \varphi_{w 3} & \varphi_{w 4} & y_{f 1} & \varphi_{f 1} & \theta_{f 1} & y_{f 2} & \varphi_{f 2} & \theta_{f 2} & y_{c} & \varphi_{c} & \theta_{c}
\end{array}\right]^{T} \text {, } \\
& \mathbf{Y}_{2}=\left[\begin{array}{llllll}
x_{d L 1} & x_{d R 1} & x_{d L 2} & x_{d R 2} & x_{y 1} & x_{y 2}
\end{array}\right]^{T} \text {. }
\end{aligned}
$$

Aerodynamic load is proportional to the square of the speed; thus, it is reasonable to consider its effect on the hunting stability of the vehicle on tracks at high speed. There have been studies on the dynamic behavior of railway vehicles that have considered the aerodynamic loads. However, nearly all such works deal with the forced vibration induced by aerodynamic load. Hardly any work concerns the influence of aerodynamic load on the self-excited vibration of railway vehicles or hunting stability. Forced vibration analysis cannot offer meaningful results to evaluate the stability of motion; hence, the existing works on the forced vibration of railway vehicle considering the aerodynamic load cannot be used in the analysis 
of the hunting stability. For example, it is well known that for linear hunting stability, critical speed is the most important parameter. However, if we use the existing methods for hunting analysis, the linear critical speed does not change regardless of how big the aerodynamic load is, because these methods do not consider the aerodynamic loads. If we use the existing methods for forced vibration, which do consider the aerodynamic load, the linear critical speed, which is an eigenvalue, cannot be obtained because the typical forced vibration analysis does not yield eigenvalues. Actually, the hunting stability will change because of the aerodynamic load. To solve the problem, a mathematical model for the hunting stability of a vehicle considering the aerodynamic load is presented in this study. The key issue, as it will be shown below, is the recognition of the change of the intrinsic parameters of the vehicle dynamics model owing to the aerodynamic load. By using the model the solution to the linear hunting stability (eigenvalue problem) of a high-speed railway vehicle considering the aerodynamic load is obtained, and the changes of the linear critical speed (eigenvalue) and the eigenvectors owing to the aerodynamic load are investigated quantitatively.

The aerodynamic load may change the important intrinsic parameters of the vehicle dynamics model, such as the wheel-rail creepage coefficients, the gravitational stiffness, and the gravitational angular stiffness of the wheelsets. These changes derive from the variation of the wheel-rail normal forces or axle loads induced by the aerodynamic load. Consequently, the hunting stability may vary as well. This is the main reason for the changes in the linear hunting stability owing to the aerodynamic load in general. In this study, the following are considered: (1) the changes in the wheel-rail creepage coefficients, the gravitational stiffness and gravitational angular stiffness owing to the aerodynamic lift force, the drag force, and the pitching moment; (2) the differences in the creepage coefficients between the left and right wheels of a wheelset because of the different wheel-rail normal forces owing to the lateral aerodynamic force and overturning moment; (3) the differences in the creepage coefficients between one wheelset and every wheelset because of the different wheel-rail normal forces owing to the aerodynamic drag force and pitching moment. The effects of the yaw moment are ignored in this study because it does not contribute to the wheel-rail normal forces. Based on all the above, the differential equation of motion for the hunting stability of a railway vehicle is

$$
\begin{gathered}
{\left[\begin{array}{cc}
\mathbf{M}_{1} & 0 \\
0 & 0
\end{array}\right]\left\{\begin{array}{c}
\ddot{\mathbf{Y}}_{1} \\
\ddot{\mathbf{Y}}_{2}
\end{array}\right\}+\left[\begin{array}{cc}
\mathbf{C}_{1}\left(C_{L}, C_{D}, C_{C}, C_{M x}, C_{M y}, \mathbf{U}, \mathbf{V}\right) & 0 \\
\mathbf{C}_{2} & \mathbf{C}_{3}
\end{array}\right]\left\{\begin{array}{c}
\dot{\mathbf{Y}}_{1} \\
\dot{\mathbf{Y}}_{2}
\end{array}\right\}} \\
+\left[\begin{array}{cc}
\mathbf{K}_{1}\left(C_{L}, C_{D}, C_{C}, C_{M x}, C_{M y}, \mathbf{U}, \mathbf{V}\right) & \mathbf{K}_{2} \\
\mathbf{K}_{3} & \mathbf{K}_{4}
\end{array}\right]\left\{\begin{array}{c}
\mathbf{Y}_{1} \\
\mathbf{Y}_{2}
\end{array}\right\}=0,
\end{gathered}
$$

where $C_{L}, C_{D}, C_{C}, C_{M x}$, and $C_{M y}$ are, respectively, the aerodynamic coefficients of lift force, drag force, lateral force, overturning moment, and pitching 
moment; $\mathbf{U}$ and $\mathbf{V}$ are, respectively, the velocity vectors of crosswind and the running speed of the vehicle; $\mathbf{M}_{1}$ is the inertia matrix; $\mathbf{C}_{1}\left(C_{L}, C_{D}, C_{C}, C_{M x}, C_{M y}\right.$, $\mathbf{U}, \mathbf{V})$ and $\mathbf{K}_{1}\left(C_{L}, C_{D}, C_{C}, C_{M x}, C_{M y}, \mathbf{U}, \mathbf{V}\right)$ are, respectively, the damping and stiffness matrices consisting of contributions from the wheel-rail creep, gravitational stiffness and angular stiffness, and the primary and secondary suspension (some elements of $\mathbf{C}_{1}$ and $\mathbf{K}_{1}$ are affected by the aerodynamic loads); $\mathbf{C}_{2}$ and $\mathbf{C}_{3}$ are the damping matrices of the lateral and yaw dampers, respectively; $\mathbf{K}_{2}, \mathbf{K}_{3}$, and $\mathbf{K}_{4}$ are the supporting stiffness matrices of the spring-damping connecting point of the lateral and yaw dampers, respectively.

To obtain the linear critical hunting speed of the vehicle considering the aerodynamic load, we analyze the eigenvalue problem for the vehicle dynamic system characterized by Eq. (8) at the equilibrium position considering the aerodynamic load in the presence of crosswind. If the real part of an eigenvalue becomes zero, the vehicle system reaches the critical state and the corresponding speed is the linear critical hunting speed.

The usual equations of motion, which are listed below for comparison, do not include the effect of aerodynamic load.

$$
\left[\begin{array}{cc}
\mathbf{M}_{1} & 0 \\
0 & 0
\end{array}\right]\left\{\begin{array}{c}
\ddot{\mathbf{Y}}_{1} \\
\ddot{\mathbf{Y}}_{2}
\end{array}\right\}+\left[\begin{array}{cc}
\mathbf{C}_{1} & 0 \\
\mathbf{C}_{2} & \mathbf{C}_{3}
\end{array}\right]\left\{\begin{array}{c}
\dot{\mathbf{Y}}_{1} \\
\dot{\mathbf{Y}}_{2}
\end{array}\right\}+\left[\begin{array}{cc}
\mathbf{K}_{1} & \mathbf{K}_{2} \\
\mathbf{K}_{3} & \mathbf{K}_{4}
\end{array}\right]\left\{\begin{array}{c}
\mathbf{Y}_{1} \\
\mathbf{Y}_{2}
\end{array}\right\}=\mathbf{0} .
$$

It is obvious that each term in Eq. (9) has nothing to do with the aerodynamic load; therefore the usual method based on Eq. (9) cannot deal with the hunting stability including the effects of the aerodynamic load. We only use Eq. (8), which we developed, for this purpose.

Because of the relative velocity between the vehicle system and air, the induced airflow creates pressure and frictional shear stress on the vehicle body surface and forms the aerodynamic drag force, lateral force, and lift force $F_{i}(i=1-3)$, overturning moment, pitching moment, and yaw moment $M_{i}(i=1-3)$. They are expressed as Eqs. (10) and (11), e.g. Refs. 29-31,

$$
\begin{gathered}
F_{i}=\frac{1}{2} \rho A C_{i}|\mathbf{V}+\mathbf{U}|^{2} \quad(i=1,2,3), \\
C_{1}=C_{D}, \quad C_{2}=C_{L}, \quad C_{3}=C_{C} . \\
M_{i}=\frac{1}{2} \rho A L C_{M i}|\mathbf{V}+\mathbf{U}|^{2} \quad(i=1,2,3), \\
C_{M 1}=C_{M x}, \quad C_{M 2}=C_{M y}, \quad C_{M 3}=C_{M z},
\end{gathered}
$$

where $\rho, A$, and $L$ are, respectively, the air density, reference area, and reference length; $C_{L}, C_{D}, C_{C}, C_{M x}, C_{M y}$, and $C_{M z}$ are the coefficients of aerodynamic lift force, drag force, lateral force, overturning moment, pitching moment, and yaw moment, respectively. 
The aerodynamic lift, drag and lateral force, overturning and pitching moment affect the wheel-rail normal forces. Omitting the high-order small quantities, the variation of the wheel-rail normal forces is obtained by Eq. (12).

$$
\begin{aligned}
& \sum_{i=1}^{4}\left(\mathbf{F}_{w R i}+\mathbf{F}_{w L i}\right)+\mathbf{F}_{C_{L}}=0 \\
& \sum_{i=1}^{4}\left(\mathbf{r}_{R i} \times \mathbf{F}_{w R i}+\mathbf{r}_{L i} \times \mathbf{F}_{w L i}\right)+\mathbf{r}_{C_{L}} \times \mathbf{F}_{C_{L}}+\mathbf{r}_{C_{D}} \times \mathbf{F}_{C_{D}}+\mathbf{r}_{C_{C}} \\
& \quad \times \mathbf{F}_{C_{C}}+\mathbf{r}_{x} \times \mathbf{F}_{x}+\mathbf{r}_{y} \times \mathbf{F}_{y}+\mathbf{M}=0
\end{aligned}
$$

where $\mathbf{F}_{w L i}, \mathbf{F}_{w R i}, \mathbf{r}_{L i}$ and $\mathbf{r}_{R i}$ are, respectively, the variation of the wheel-rail normal forces of the left and right wheel of a wheelset and their position vectors; $\mathbf{F}_{C L}$ and $\mathbf{r}_{C L}$ are the aerodynamic lift load and its position vector; $\mathbf{F}_{C D}$ and $\mathbf{r}_{C D}$ are the aerodynamic drag force and its position vector; $\mathbf{F}_{C C}$ and $\mathbf{r}_{C C}$ are the aerodynamic lateral force and its position vector; $\mathbf{F}_{x}$ and $\mathbf{r}_{x}$ are the traction (counteracting the drag) and its radius vector; $\mathbf{F}_{y}$ and $\mathbf{r}_{y}$ are the wheel-rail lateral force and its radius vector; $\mathbf{M}$ is the moment vector.

Incorporating the variation of the wheel-rail normal forces obtained by Eq. (12), the changed wheel-rail normal forces after considering the aerodynamic loads, $N_{L i}$ and $N_{R i}$, of the left and right wheels of a wheelset are

$$
\begin{aligned}
& N_{L i}=\frac{W}{2}-\frac{1}{16} \rho A\left(C_{L}+\frac{Z_{O A} C_{C}}{d_{0}} \pm \frac{Z_{O A} C_{D}}{l_{c}}+\frac{L C_{M x}}{l_{c}} \pm \frac{L C_{M y}}{d_{0}}\right)|\mathbf{V}+\mathbf{U}|^{2} \\
& N_{R i}=\frac{W}{2}-\frac{1}{16} \rho A\left(C_{L}-\frac{Z_{O A} C_{C}}{d_{0}} \pm \frac{Z_{O A} C_{D}}{l_{c}}-\frac{L C_{M x}}{l_{c}} \pm \frac{L C_{M y}}{d_{0}}\right)|\mathbf{V}+\mathbf{U}|^{2}
\end{aligned}
$$

$$
(i=1,2,3,4)
$$

Then, the changed gravitational stiffness $K_{g i}$ and the gravitational angular stiffness $C_{g i}$ after considering the aerodynamic loads are

$$
\begin{aligned}
K_{g i} & =\left(W-\frac{1}{8} \rho A\left(C_{L} \pm \frac{Z_{O A} C_{D}}{l_{c}} \pm \frac{L C_{M y}}{l_{c}}\right)|\mathbf{V}+\mathbf{U}|^{2}\right) \frac{\lambda}{b} \\
C_{g i} & =\left(W-\frac{1}{8} \rho A\left(C_{L} \pm \frac{Z_{O A} C_{D}}{l_{c}} \pm \frac{L C_{M y}}{l_{c}}\right)|\mathbf{V}+\mathbf{U}|^{2}\right) \lambda b
\end{aligned}
$$

where $W, \lambda, b, Z_{O A}$, and $l_{c}$ are the axle load, wheel conicity, half of the track gauge, the vertical distance from the aerodynamic force application point to the track, and half of the bogie center distance.

Similarly, the changed creepage coefficients owing to aerodynamic load are obtained by substituting the wheel-rail normal forces in Eq. (13) for the usual normal forces without considering the aerodynamic load. According to Kalker's 
linear theory, ${ }^{32}$ the changed creep force coefficients $f_{11}$ and $f_{22}$ after considering the aerodynamic load are

$$
\begin{aligned}
f_{i i L}= & G m n\left[\frac{3 \pi\left(1-\sigma^{2}\right)}{2 E\left(A_{f}+B_{f}\right)}\right]^{\frac{2}{3}} \\
& \times\left[\frac{W}{2}-\frac{1}{16} \rho A\left(C_{L}+\frac{Z_{O A} C_{C}}{d_{0}} \pm \frac{Z_{O A} C_{D}}{l_{c}}+\frac{L C_{M x}}{l_{c}} \pm \frac{L C_{M y}}{d_{0}}\right)|\mathbf{V}+\mathbf{U}|^{2}\right]^{\frac{2}{3}} C_{i i}, \\
f_{i i R}= & G m n\left[\frac{3 \pi\left(1-\sigma^{2}\right)}{2 E\left(A_{f}+B_{f}\right)}\right]^{\frac{2}{3}} \\
& \times\left[\frac{W}{2}-\frac{1}{16} \rho A\left(C_{L}-\frac{Z_{O A} C_{C}}{d_{0}} \pm \frac{Z_{O A} C_{D}}{l_{c}}-\frac{L C_{M x}}{l_{c}} \pm \frac{L C_{M y}}{d_{0}}\right)|\mathbf{V}+\mathbf{U}|^{2}\right]^{\frac{2}{3}} C_{i i}, \\
& (i=1,2)
\end{aligned}
$$

where

$$
\left(A_{f}+B_{f}\right)=\frac{1}{2}\left(\frac{1}{R_{w}}+\frac{1}{r_{r}}+\frac{1}{r_{w}}\right)
$$

and $R_{w}, r_{w}$, and $r_{r}$ are, respectively, the radius of the wheel, the radius of the wheel tread, and the rail profile; $G$ and $E$ are the shear modulus and Young's modulus; $\sigma$ is the Poisson's ratio; $m$ and $n$ are coefficients that depend on $A_{f}$ and $B_{f}$; and $C_{i i}$ is a dimensionless coefficient. ${ }^{32}$

The usual expressions for the gravitational stiffness, the gravitational angular stiffness, and the creepage coefficients do not consider the aerodynamic load and are listed for comparison below.

$$
\begin{gathered}
K_{g i}=W \frac{\lambda}{b}, \quad C_{g i}=W \lambda b \quad(i=1,2,3,4), \\
f_{11}=G m n\left(\frac{3 \pi W\left(1-\sigma^{2}\right)}{4 E\left(A_{f}+B_{f}\right)}\right)^{\frac{2}{3}} C_{11}, \quad f_{22}=G m n\left(\frac{3 \pi W\left(1-\sigma^{2}\right)}{4 E\left(A_{f}+B_{f}\right)}\right)^{\frac{2}{3}} C_{22} .
\end{gathered}
$$

The terms in Eqs. (17) and (18) have nothing to do with the aerodynamic load; therefore, the method based on Eqs. (17) and (18) cannot deal with the hunting stability including the effects of the aerodynamic load. To tackle this, Eqs. (13)-(15) should be used for applications involving changed wheel-rail normal forces, gravitational stiffness, gravitational angular stiffness and creepage coefficients. This clearly makes the hunting behavior of high-speed vehicles different from their low-speed counterparts. 


\section{Numerical Results and Discussion}

To describe the method for calculating the numerical results, Eq. (8) is rewritten as follows:

$$
\mathbf{M} \ddot{\mathbf{Y}}+\mathbf{C}\left(C_{L}, C_{D}, C_{C}, C_{M x}, C_{M y}, \mathbf{U}, \mathbf{V}\right) \dot{\mathbf{Y}}+\mathbf{K}\left(C_{L}, C_{D}, C_{C}, C_{M x}, C_{M y}, \mathbf{U}, \mathbf{V}\right) \mathbf{Y}=\mathbf{0}
$$

where $\mathbf{M}, \mathbf{C}, \mathbf{K}$ and $\mathbf{Y}$ are the mass matrix, damping matrix, stiffness matrix, and displacement vector, respectively. Furthermore, a new vector $\mathbf{Z}$ is defined as

$$
\mathbf{Z}=\left[\begin{array}{ll}
\dot{\mathbf{Y}} & \mathbf{Y}
\end{array}\right]^{T} \text {. }
$$

Then, Eq. (19) becomes

$$
\dot{\mathbf{Z}}=\mathbf{A Z},
$$

which is the standard form readily solved for the eigenvalue problem.

$$
\mathbf{A}=\left[\begin{array}{cc}
-\mathbf{M}^{-1} \mathbf{C}\left(C_{L}, C_{D}, C_{C}, C_{M x}, C_{M y}, \mathbf{U}, \mathbf{V}\right) & -\mathbf{M}^{-1} \mathbf{K}\left(C_{L}, C_{D}, C_{C}, C_{M x}, C_{M y}, \mathbf{U}, \mathbf{V}\right) \\
\mathbf{I} & \mathbf{O}
\end{array}\right],
$$

where $\mathbf{I}$ and $\mathbf{O}$ are the identity and zero matrices.

If the real parts of all eigenvalues of matrix $\mathbf{A}$ are negative, the vehicle system is stable. If a real part of the eigenvalue of matrix $\mathbf{A}$ is positive, the system is unstable. If the maximum real part of the eigenvalue is zero, the system is at the critical state, and the corresponding minimum speed is the linear critical speed.

Examples of the critical hunting speed for railway vehicles on straight tracks were presented in Ref. 15, in which the aerodynamic load was not considered. These examples were recalculated as degenerated cases for verification by using our computer program capable of considering the effects of the aerodynamic load. The linear critical speed versus the conicity of wheel tread is shown in Fig. 2, where the maximum deviation between the results of this study and Ref. 15 is less than $1 \%$.

We can see that the damping and stiffness matrices $\mathbf{C}$ and $\mathbf{K}$ in Eq. (19) are independent of the response vectors, such as displacement, velocity, acceleration vectors; therefore Eq. (19) is the linear equation suitable for the eigenvalue analysis. If the wheel-rail contact is nonlinear or a nonlinear suspension system is considered, matrices $\mathbf{C}$ and $\mathbf{K}$ in Eq. (19) depend on the response vectors. Then, matrices $\mathbf{C}$ and $\mathbf{K}$ become $\mathbf{C}\left(C_{L}, C_{D}, C_{C}, C_{M x}, C_{M y}, \mathbf{U}, \mathbf{V}, \mathbf{Y}, \dot{\mathbf{Y}}\right)$ and $\mathbf{K}\left(C_{L}, C_{D}, C_{C}, C_{M x}, C_{M y}, \mathbf{U}, \mathbf{V}, \mathbf{Y}, \dot{\mathbf{Y}}\right)$, and Eq. (19) is a nonlinear equation. In this case, the nonlinear critical speed should be calculated. ${ }^{33}$ In this study, we mainly focus on the eigenvalue analyses for the linearized model to understand the essential features of the high-speed railway vehicle system, including the effects of the aerodynamic loads. Regarding hunting stability, the eigenvalue analysis and nonlinear analysis are complementary; details for the latter are given in Ref. 33. Several results 


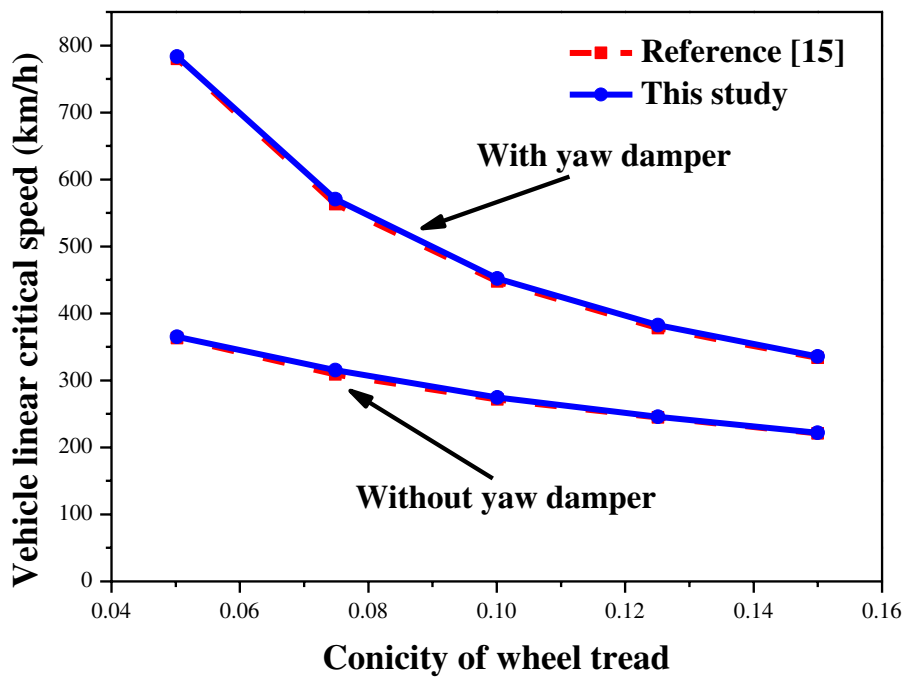

Fig. 2. Comparison of the linear critical velocity of a railway vehicle.

Table 1. Nonlinear critical speed.

\begin{tabular}{lc}
\hline Aerodynamic condition & Nonlinear critical speed $(\mathrm{km} / \mathrm{h})$ \\
\hline Without aerodynamic load & 377 \\
With aerodynamic load (crosswind velocity $5.4 \mathrm{~m} / \mathrm{s})$ & 385 \\
With aerodynamic load (crosswind velocity $10.7 \mathrm{~m} / \mathrm{s})$ & 351 \\
With aerodynamic load (crosswind velocity $15.0 \mathrm{~m} / \mathrm{s})$ & 242 \\
\hline
\end{tabular}

for the nonlinear critical speed are briefly given in Table 1 . The effects of the joint actions of the aerodynamic load and other parameters on the nonlinear critical speed are complex, and details are given in Ref. 33 and thus omitted here. Except for Table 1, the rest of this study only concerns about the eigenvalue analysis.

\subsection{The effect of the aerodynamic load on linear critical speed}

The effect of the steady aerodynamic load, from the actions of the crosswind and airflow in the opposite direction of the train, on the linear critical hunting speed of the railway vehicle is discussed in this section. Numerical calculations were performed for a typical high-speed railway vehicle. The parameters of the vehicle system are given in the appendix. The eigenvalues corresponding to the hunting instability mode of the vehicle were calculated for four different cases, three of which incorporate the effect of the aerodynamic load (crosswind velocities of 5.40, 10.7 and $15.0 \mathrm{~m} / \mathrm{s}$, respectively). The results are shown in Fig. 3. The vehicle running speed corresponding to the zero-real part of the eigenvalue is the linear critical speed. It is clearly seen that the aerodynamic load can decrease the linear critical speed. 


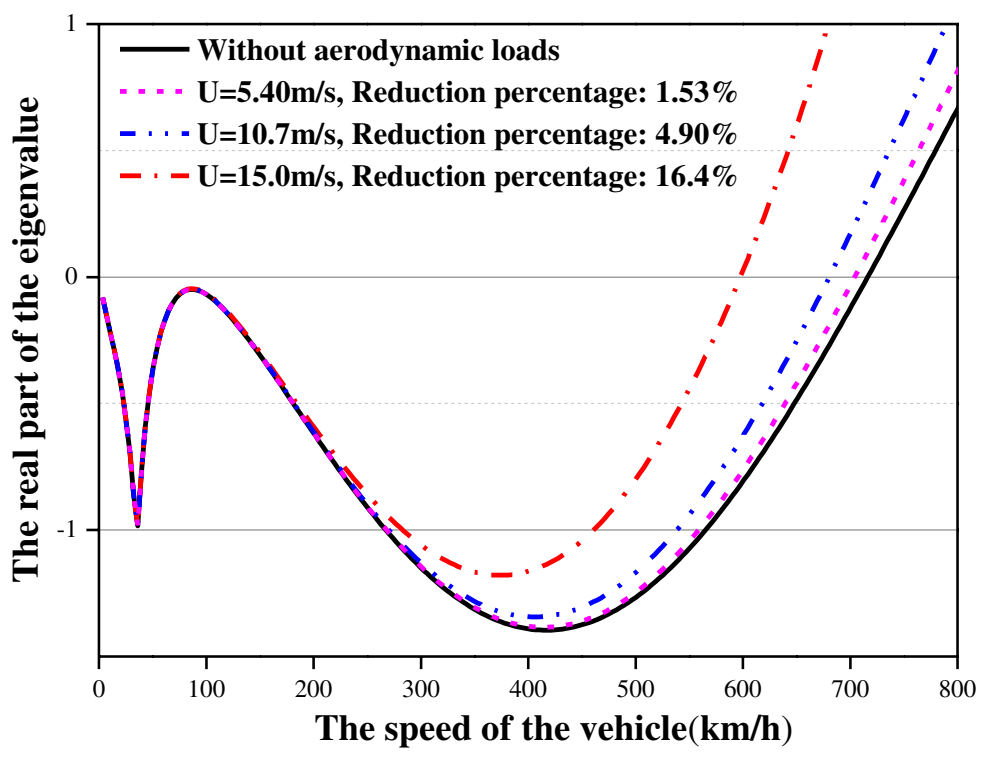

Fig. 3. Real part of the eigenvalues of the instability mode.

The linear critical speed decreases monotonously as the velocity of the beam wind increases. For the example considered in this study, the linear critical speed incorporating the effect of the aerodynamic load is 704,680 and $598 \mathrm{~km} / \mathrm{h}$, which is $1.53 \%$, $4.90 \%$ and $16.4 \%$ less than the linear critical speed without the effect of the aerodynamic load. For the three cases, the beam wind velocities are 5.40, 10.7 and $15.0 \mathrm{~m} / \mathrm{s}$. Figure 4 is the root locus diagram showing the loci of the eigenvalues as the running speed of the vehicle increases beyond the linear critical speed. Lines (1), (2), and (3) in Fig. 4 are lines connecting the points of equal running speeds in different aerodynamic cases. All running speeds on line (1) (line (2) or line (3) correspond to a same speed - the linear critical speed for the case of the crosswind velocities being $5.40 \mathrm{~m} / \mathrm{s}(10.7 \mathrm{~m} / \mathrm{s}$ or $15.0 \mathrm{~m} / \mathrm{s})$. It is obvious that for the same running speed, the real part of the eigenvalue increases with the increasing crosswind velocity, which means that the high crosswind velocity will make the railway vehicle prone to hunting.

Furthermore, to distinguish the importance of different components of the aerodynamic load, the linear critical speed was calculated for various cases where each component of the aerodynamic forces and moments is considered either individually or collectively. The numerical results are given in Table 2. It is seen that the effect of the pitching moment and lift force on the linear critical speed is stronger than that of the other components of the aerodynamic load.

The aerodynamic coefficients were provided by the aerodynamics research group directed by Prof. G. W. Yang at the Institute of Mechanics, Chinese Academy of Sciences. 


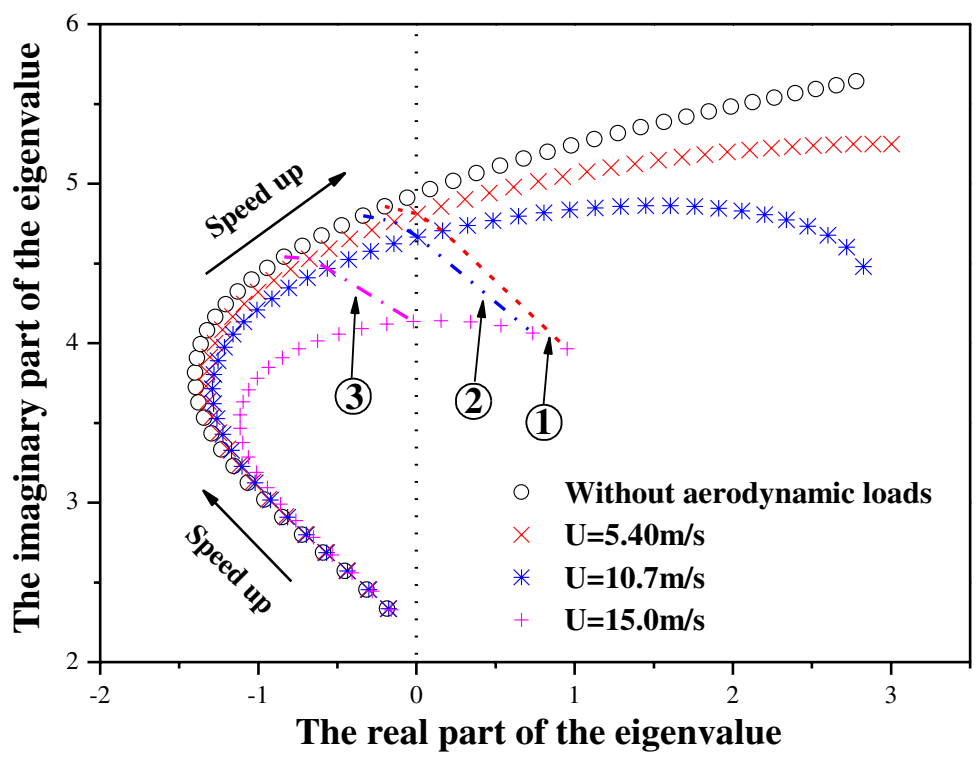

Fig. 4. Loci of the eigenvalues as the vehicle running speed increases beyond the linear critical speed.

To further understand the effect of the aerodynamic load, the values for aerodynamic load, gravitational stiffness $K_{g 1}$, gravitational angular stiffness $C_{g 1}$, creepage coefficients $f_{11 L 1}$ and $f_{11 R 1}$ for the cases where each component of the aerodynamic forces and moments is considered individually or collectively are calculated using Eqs. (10)-(15). The results for $K_{g 1}$ and $C_{g 1}$ for the vehicle speed of $600 \mathrm{~km} / \mathrm{h}$ are listed in Table 3 . The gravitational stiffness $K_{g 1}$ is $26188.9 \mathrm{~N} / \mathrm{m}$ and the gravitational angular stiffness $C_{g 1}$ is $14594.1 \mathrm{~N} \cdot \mathrm{m} / \mathrm{rad}$ when not considering the aerodynamic loads. Similarly, the results for $f_{11 L 1}$ and $f_{11 R 1}$ for the vehicle speed of $600 \mathrm{~km} / \mathrm{h}$ are listed in Table $4 ; f_{11 L 1}=f_{11 R 1}=1.373 \times 10^{7} \mathrm{~N}$ when not considering the aerodynamic loads. It is obvious that the aerodynamic load affects the gravitational stiffness, gravitational angular stiffness, and creepage coefficients. These factors are critical to a vehicle system. Therefore, the linear hunting stability is also affected.

\subsection{The effect of the aerodynamic load on instability mode}

The effect of the aerodynamic load on the instability mode is discussed below.

First, the effects of the individual components of the aerodynamic loads were investigated. For crosswind velocity of $15.0 \mathrm{~m} / \mathrm{s}$, with each component of aerodynamic forces and moments considered individually, the phasor diagram for the eigenvectors corresponding to the instability mode is shown in Fig. 5. It is obvious that, for different components of aerodynamic load acting individually or without the aerodynamic load, the complex moduli and arguments of each eigenvector element are different. To show this more clearly, the complex moduli and arguments of the 


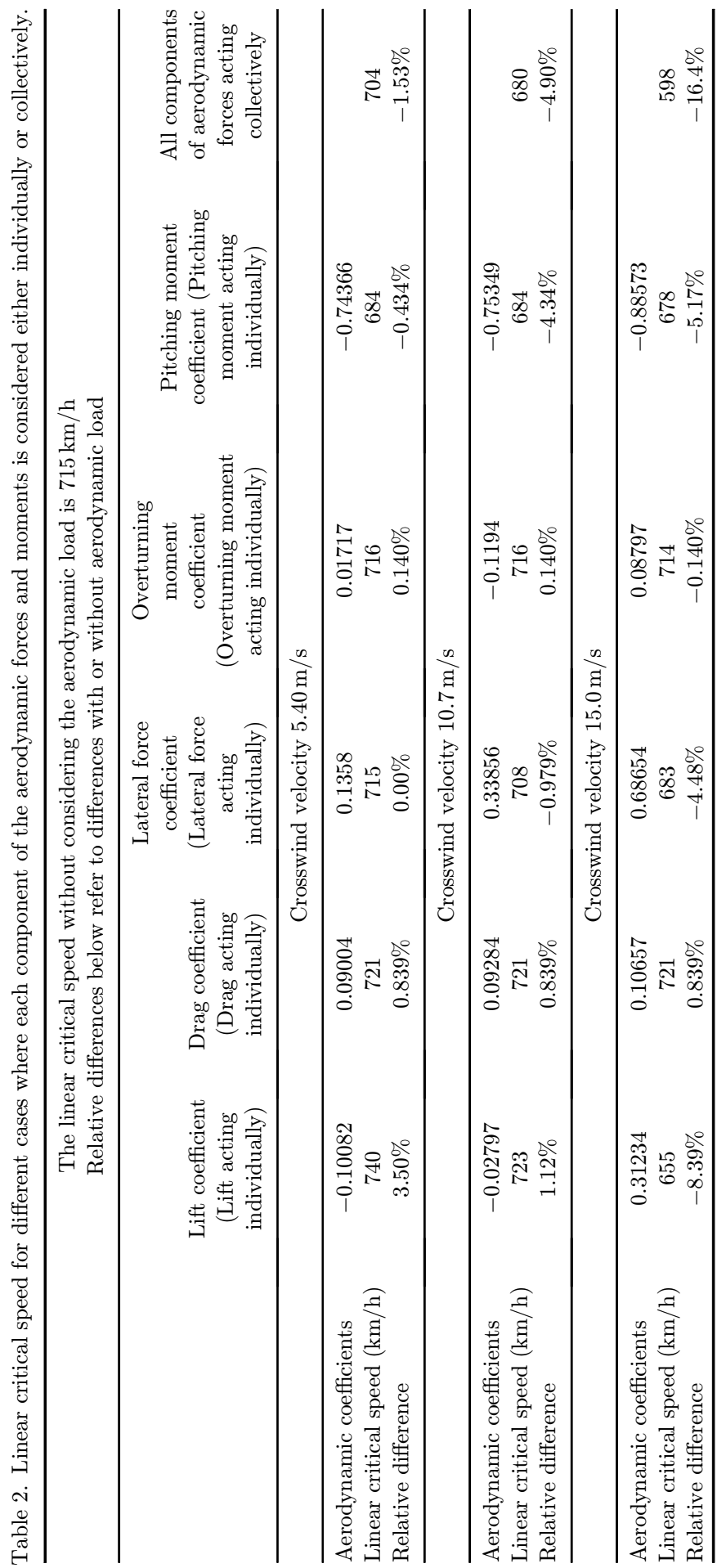




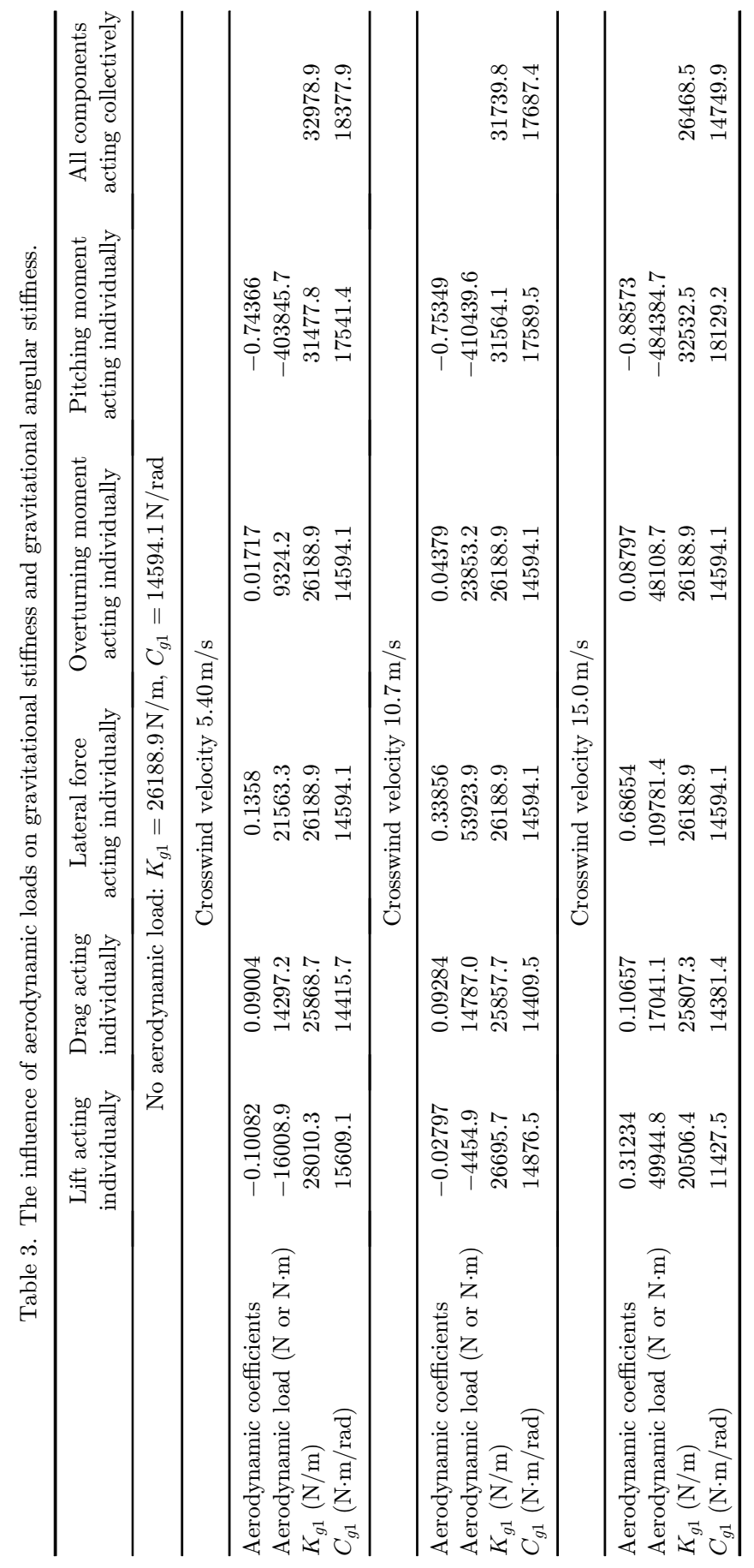




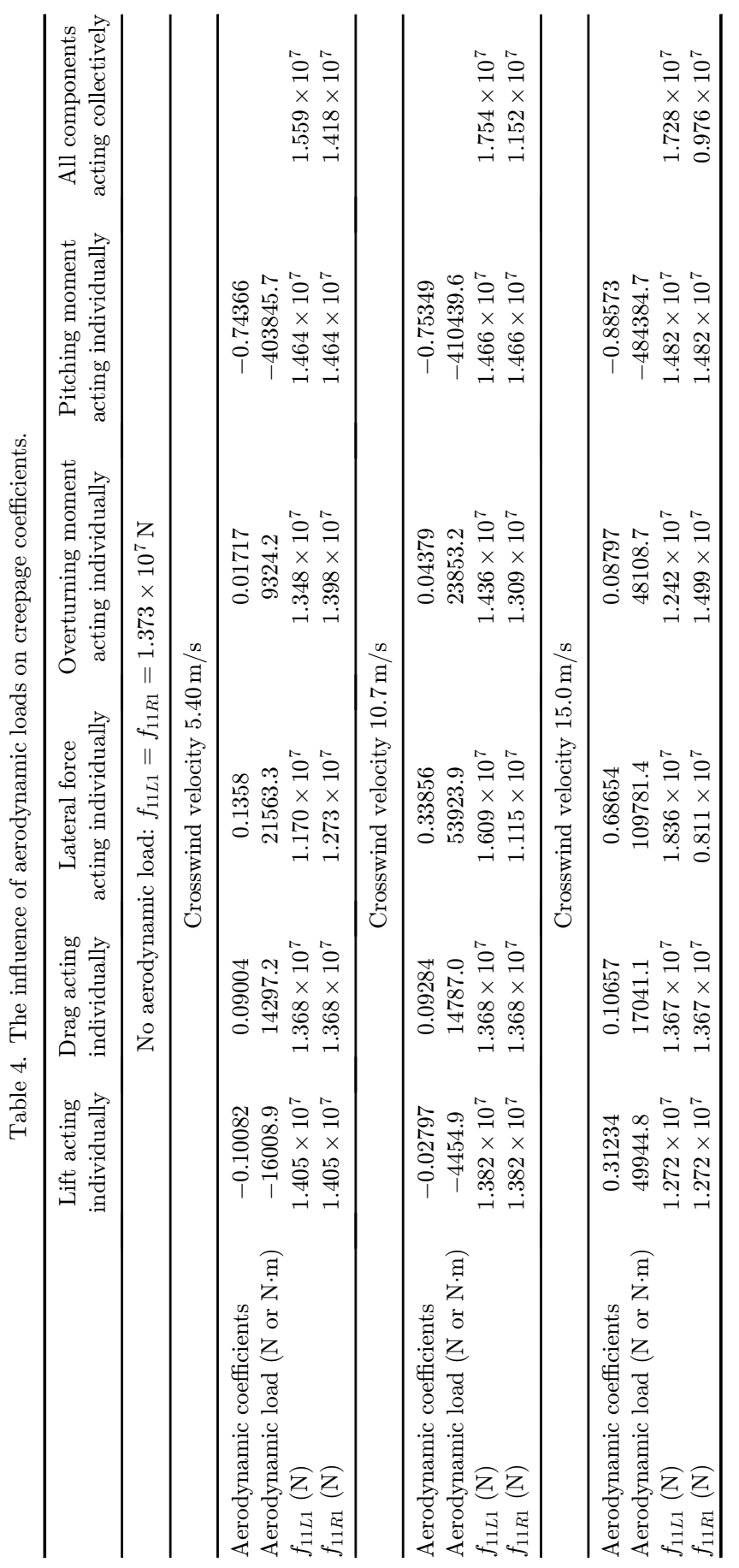




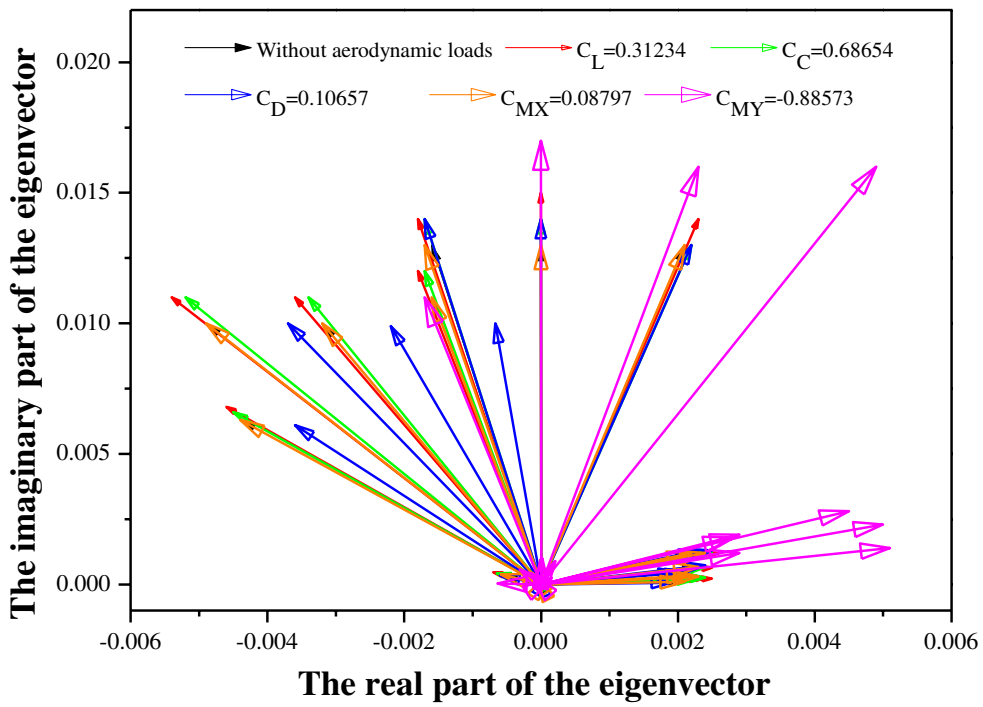

Fig. 5. Phasor diagram for the eigenvectors of the instability mode.

displacement components of the instability mode are shown separately in Figs. 6 and 7. The abscissas of Figs. 6 and 7 represent the sequence number of the first 17 elements (which describe the displacements of the vehicle system) of the eigenvectors. Every number (1-17) on the horizontal axes of Figs. 6 and 7 correspond to the displacement component of the instability mode. Numbers 1-4 correspond to the lateral displacements of the wheelset, numbers 5-8 correspond to the yaw angle of the wheelset, numbers 9 and 12 correspond to the lateral displacement of the bogie

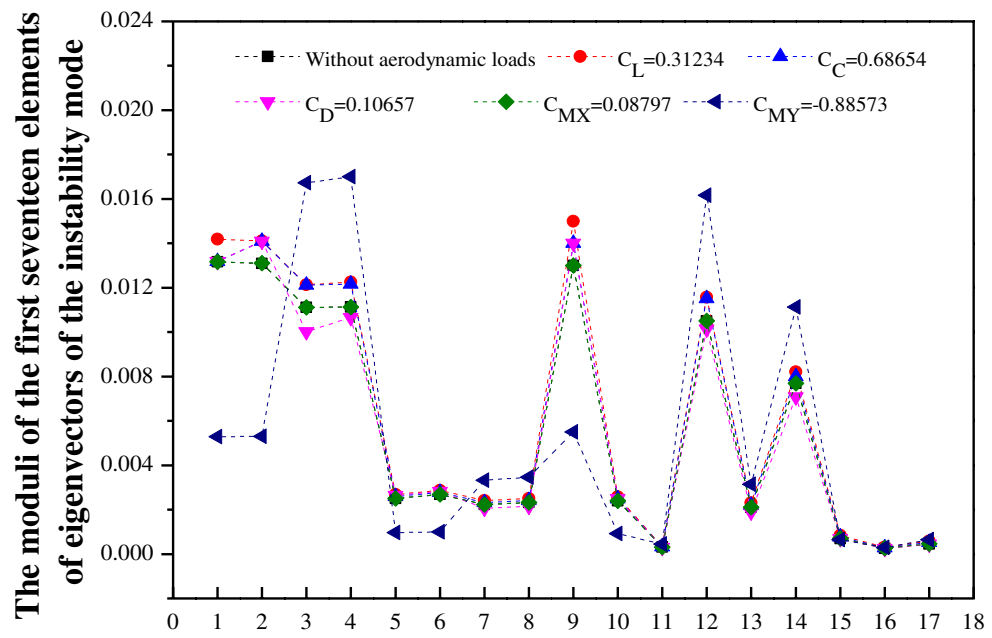

The number of the first seventeen elements of eigenvectors

Fig. 6. Moduli of the first 17 eigenvector elements of the instability mode. 


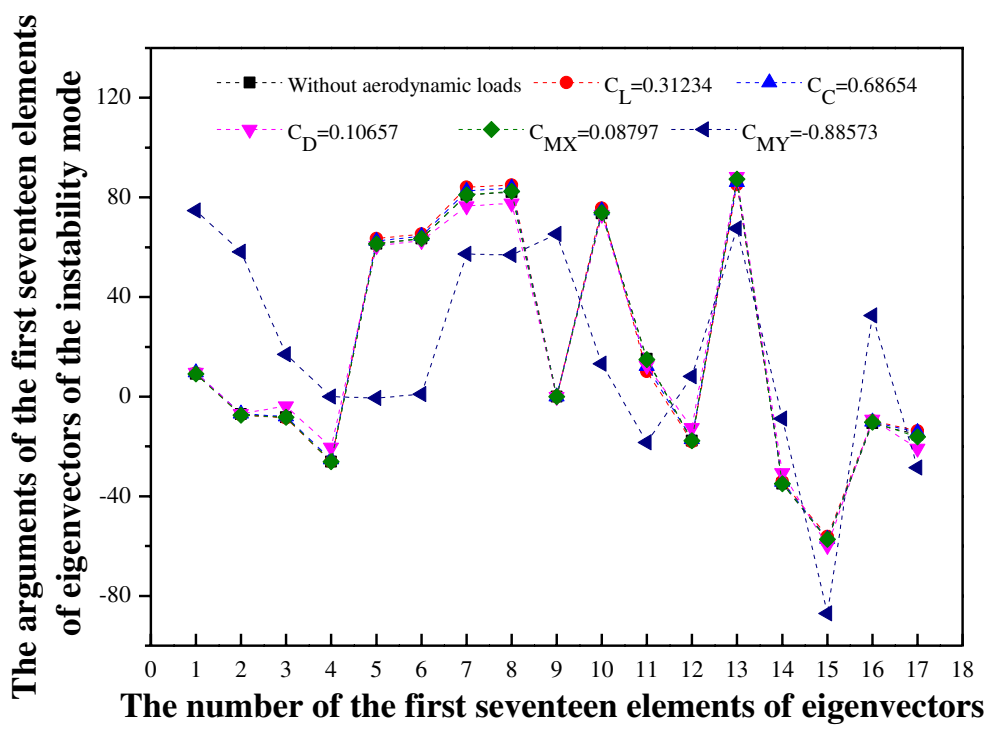

Fig. 7. Arguments of the first 17 eigenvector elements of the instability mode.

frames, numbers 10 and 13 correspond to the yaw angle of the bogie frame, numbers 11 and 14 correspond to the roll angle of the bogie frame, and numbers 15-17 correspond to the lateral displacement, and yaw and roll angles of the car body.

As shown in Figs. 6 and 7, the aerodynamic lift force, the drag force, the lateral force, and the overturning moment affect the moduli of the displacement element in the instability mode, but not the arguments. The pitching moment affects both the moduli and arguments of the displacement elements in the instability mode.

Second, we investigated the collective effect of every component of the aerodynamic load. The first 17 eigenvector elements in the instability mode are listed in Table 5 and shown in Figs. 8-12. For the four different aerodynamic load cases three involve the collective effects of all components at different crosswind velocities and the other does not consider the aerodynamic loads - the eigenvalues and eigenvectors are obviously different. By using the data in Table 5, the successive positions of the first wheelset and the front bogie during a period of time in the hunting mode (instability mode), as the vehicle moves along the track, are shown in Figs. 8 and 9. In these two figures, black denotes the case without the aerodynamic load. Blue, green, and red denote, respectively, the cases involving the collective effects of all the components of the aerodynamic loads at crosswind velocities of 5.40, 10.7 and $15.0 \mathrm{~m} / \mathrm{s}$. Clearly, the natural period of the hunting motion visibly increases with the increasing crosswind velocity. The attitudes of the wheelset and the bogie are notably changed by the actions of the aerodynamic loads.

For crosswind velocities of $5.40,10.7$ and $15.0 \mathrm{~m} / \mathrm{s}$, with all components of the aerodynamic forces and moments considered collectively, the phasor diagram for the eigenvectors corresponding to the instability mode are shown in Fig. 10. The 


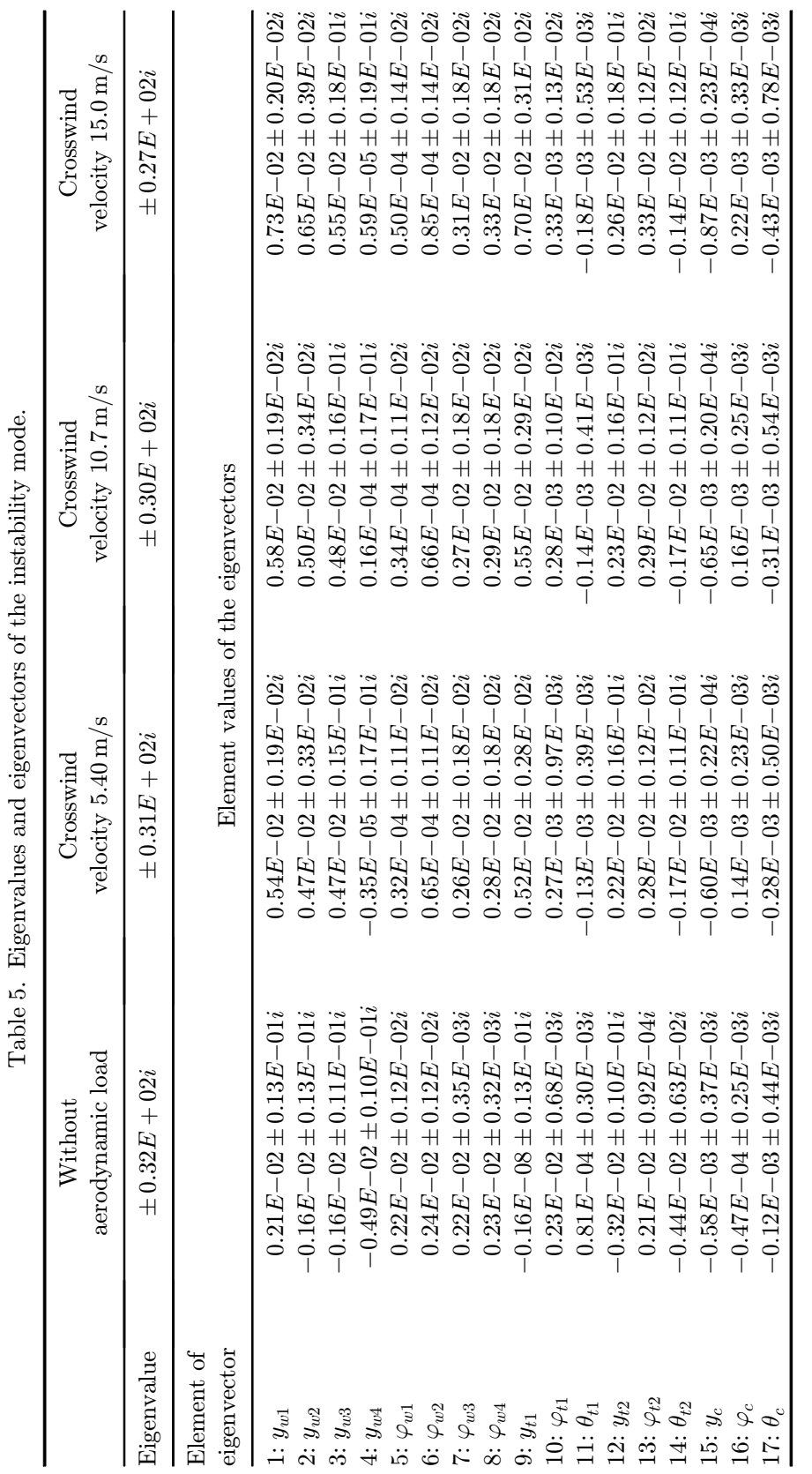




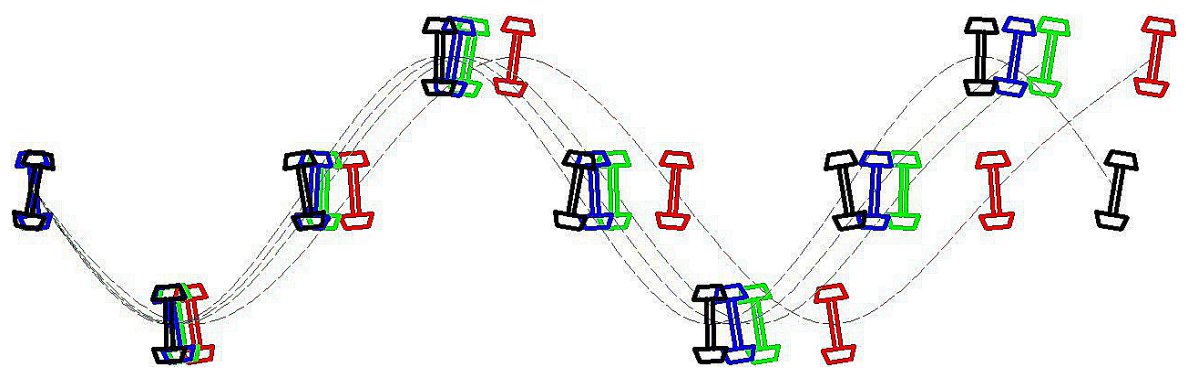

Fig. 8. Successive positions of the first wheelset in the hunting mode.

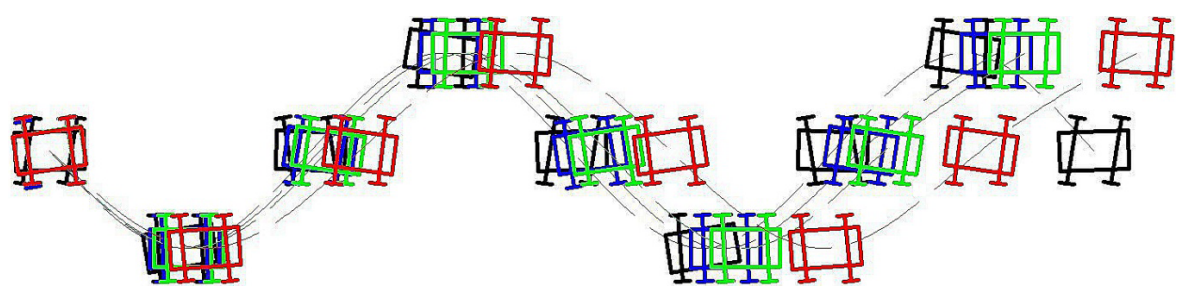

Fig. 9. Successive positions of the front bogie in the hunting mode.

eigenvectors for with or without the effect of the aerodynamic load and crosswind velocity of $5.40 \mathrm{~m} / \mathrm{s}$ are shown in Fig. 10(a), for $10.7 \mathrm{~m} / \mathrm{s}$ are shown in Fig. 10(b), and for $15.0 \mathrm{~m} / \mathrm{s}$ are shown in Fig. 10(c). The aerodynamic load apparently affects the moduli and arguments of the elements of the eigenvectors and the phase differences among the elements. These effects are clearer with the increasing crosswind velocity, as shown in Fig. 10(d). The complex moduli and arguments of the displacement components of the instability mode are shown in Figs. 11 and 12. The abscissas are the same as in Figs. 6 and 7. It is seen from Figs. 11 and 12 that the

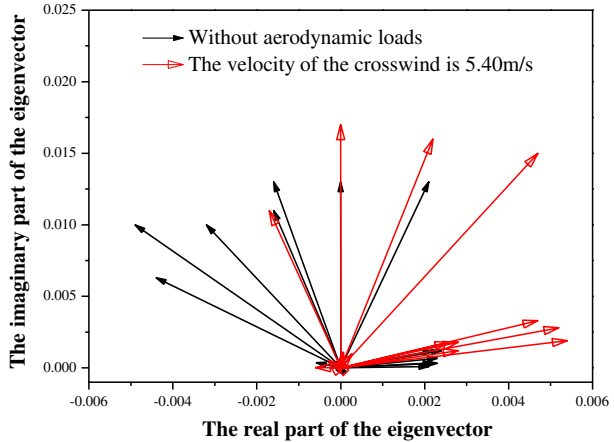

(a)

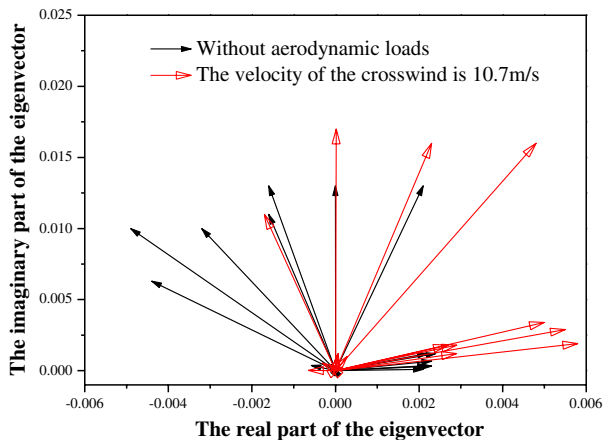

(b)

Fig. 10. Phasor diagram for the eigenvectors of the instability mode for crosswind velocity of (a) $5.4 \mathrm{~m} / \mathrm{s}$, (b) $10.7 \mathrm{~m} / \mathrm{s}$, (c) $15 \mathrm{~m} / \mathrm{s}$ and (d) all three. 


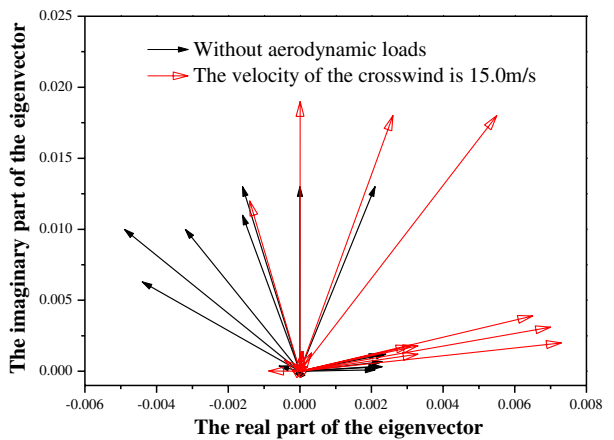

(c)

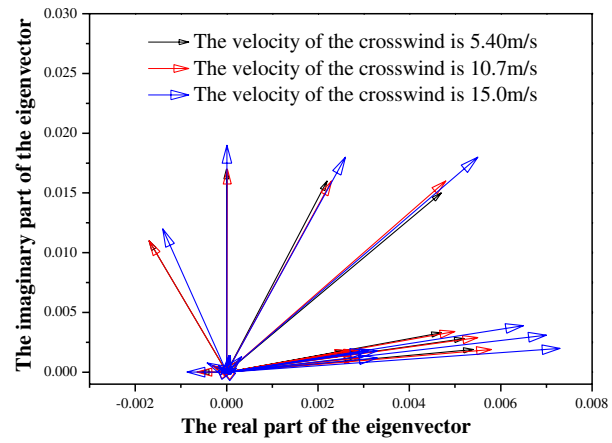

(d)

Fig. 10. (Continued)

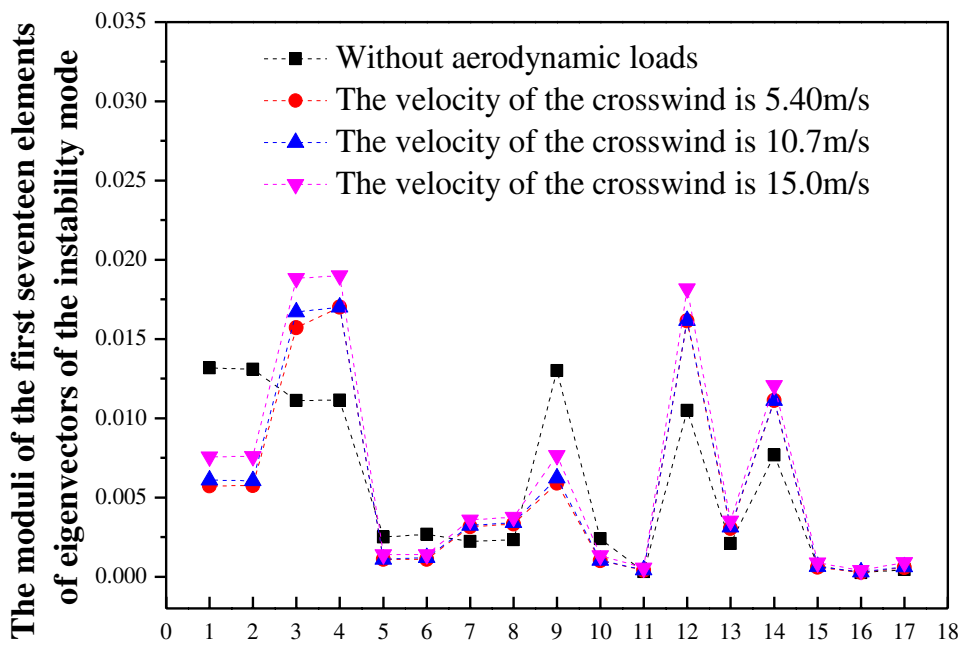

The number of the first seventeen elements of eigenvectors

Fig. 11. Moduli of the elements of eigenvectors of the instability mode at different aerodynamic conditions.

moduli and arguments of the of vehicle displacement change if the aerodynamic load is considered. The changes in the moduli of the lateral displacements of the wheelsets and bogie frames are clearer and the changes in the moduli of the displacements of the car body are small. The moduli of the lateral displacement of the rear bogie frame and its two wheelsets increase when the aerodynamic load is considered and increase monotonically with the increasing crosswind velocity; the highest increase is $70.6 \%$ for this example. The moduli of the lateral displacement of the front bogie frame and its wheelsets decrease and the highest decrease is $56.2 \%$ for this example. The changes in the arguments of the 17 displacements are all noticeable when the 


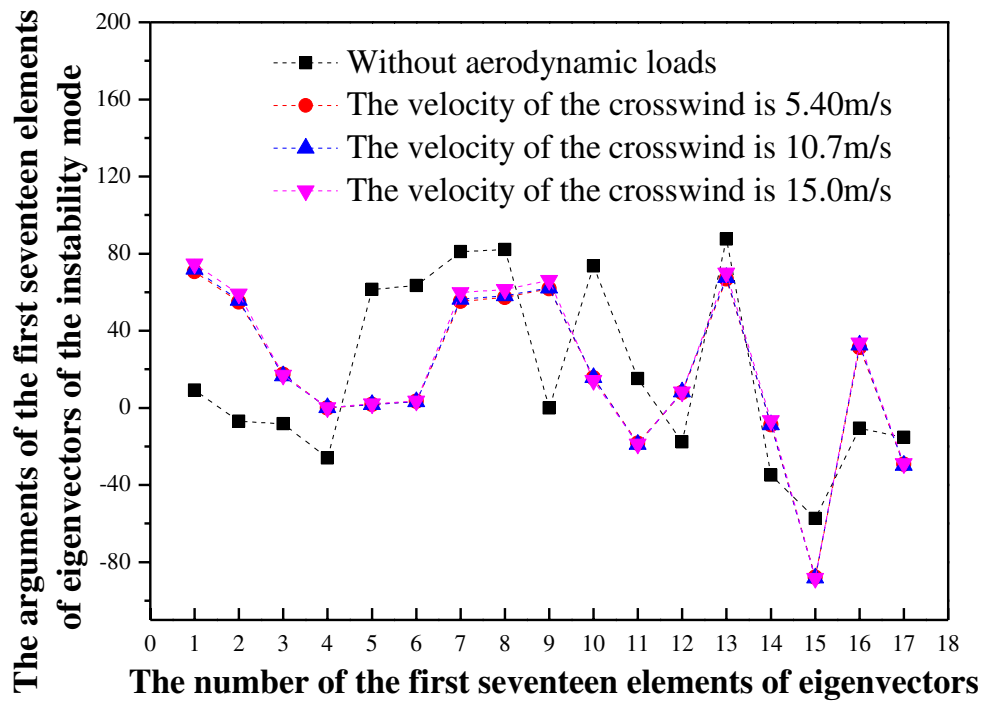

Fig. 12. Arguments of the elements of eigenvectors of the instability mode at different aerodynamic conditions.

aerodynamic load is considered, but it is difficult to differentiate among the three cases with different crosswind velocities.

\subsection{The relation between linear critical speed and suspension parameters when the aerodynamic load is considered}

With consideration of the aerodynamic load, the variation relationships between the linear critical speed and the primary/secondary suspension are presented in this section. The suspension parameters are the longitudinal and lateral stiffness of the primary suspension, and the supporting stiffness of the lateral and yaw dampers of the secondary suspension. Numerical calculations were performed at the same four aerodynamic conditions, as in preceding sections. The results are shown in Figs. 13-15.

The relation between the linear critical speed and primary suspension parameters is shown in Figs. 13(a) and 14(a). The aerodynamic load causes the linear critical speed to decrease at any stiffness of the primary suspension in the concerned range, but does not affect the variation trend of the curves of speed with respect to the stiffness of the primary suspension. For any given stiffness, the linear critical speed decreases with the increasing crosswind velocity. The speed differences for with or without the aerodynamic load are shown in Figs. 13(b) and 14(b). Figure 13(b) shows that for the lateral stiffness, the curve of speed difference is parallel to the abscissa for different crosswind velocities if the lateral stiffness is greater than a certain value, e.g. $6 \mathrm{MN} / \mathrm{m}$; that is, the speed difference does not vary for large lateral stiffness. This means that for large lateral stiffness, the interaction between the aerodynamic load and lateral stiffness is insignificant. However, the interaction is 


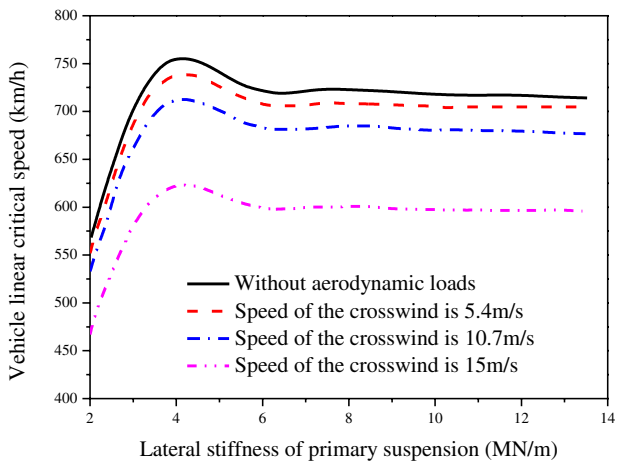

(a)

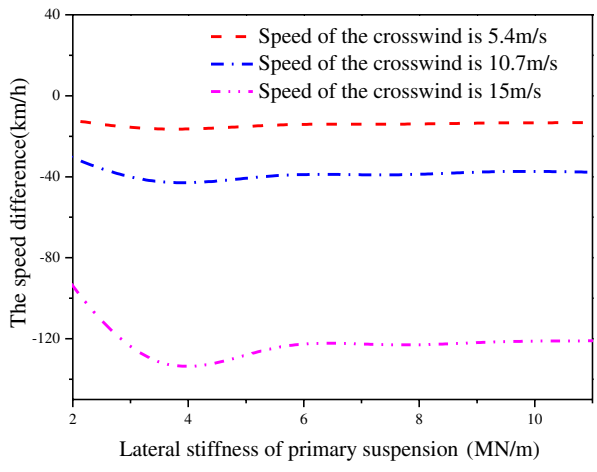

(b)

Fig. 13. (a) Linear critical speed versus lateral stiffness of primary suspension and (b) speed difference versus lateral stiffness of primary suspension.

significant for small lateral stiffness. The interaction between the aerodynamic load and lateral stiffness becomes more visible with the increasing crosswind velocity. For the longitudinal stiffness in Fig. 14(b), the interaction between the aerodynamic load and longitudinal stiffness is significant in the entire range and becomes more visible as the velocity of crosswind increases.

The relation between the linear critical speed and secondary suspension parameters is shown in Figs. 15(a) and 16(a). The aerodynamic load changes the linear critical speed for any supporting stiffness of the lateral and yaw dampers in the concerned range but does not affect the variation trend of the curves of speed with respect to the supporting stiffness of the damper. For most stiffness values, the linear critical speed decreases with the increasing crosswind velocity. The speed differences are shown in Figs. 15(b) and 16(b). For the supporting stiffness of the lateral damper, the curve of speed difference is approximately parallel to the abscissa if the

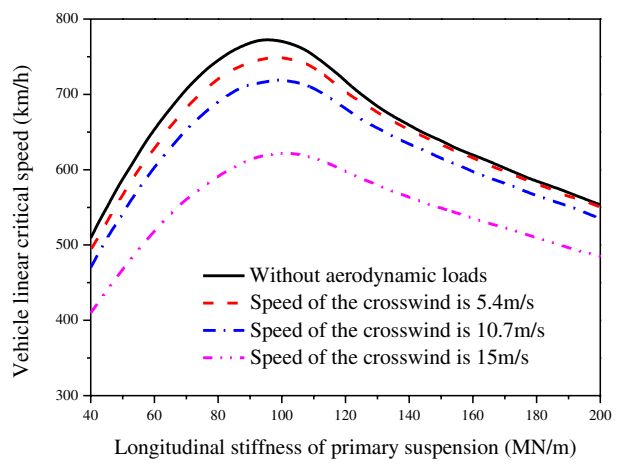

(a)

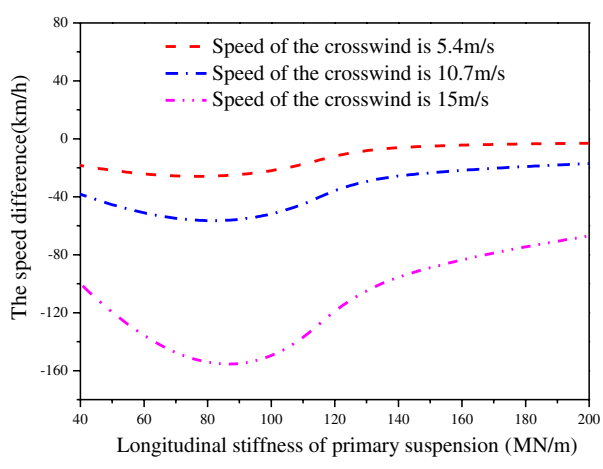

(b)

Fig. 14. (a) Linear critical speed versus longitudinal stiffness of primary suspension and (b) speed difference versus longitudinal stiffness of primary suspension. 


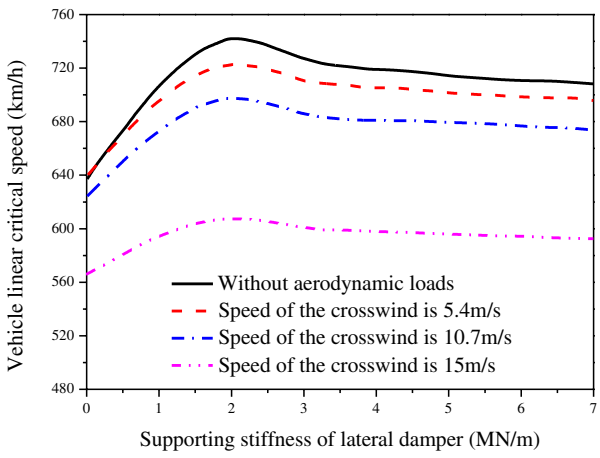

(a)

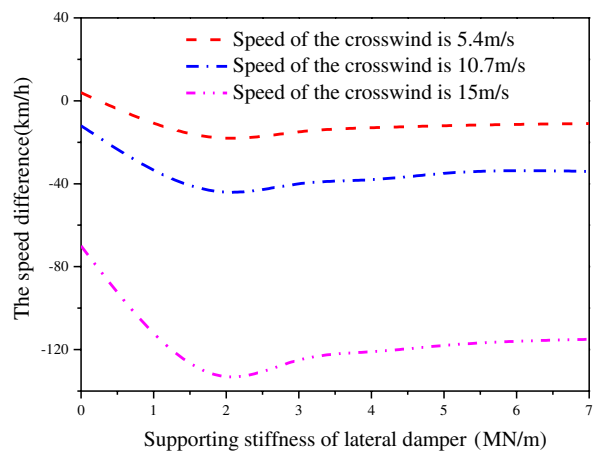

(b)

Fig. 15. (a) Linear critical speed versus supporting stiffness of lateral damper and (b) speed differences versus supporting stiffness of lateral damper.

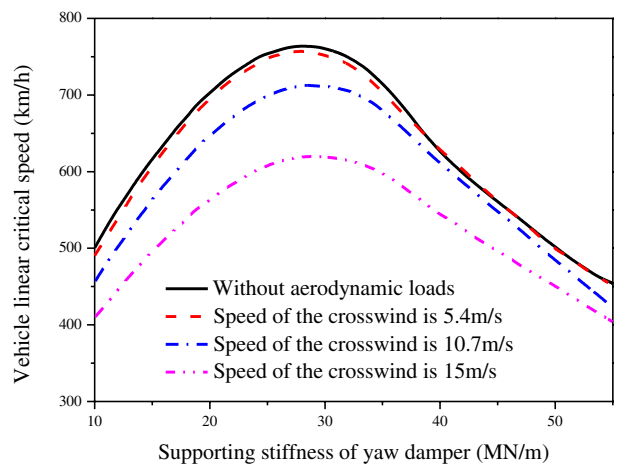

(a)

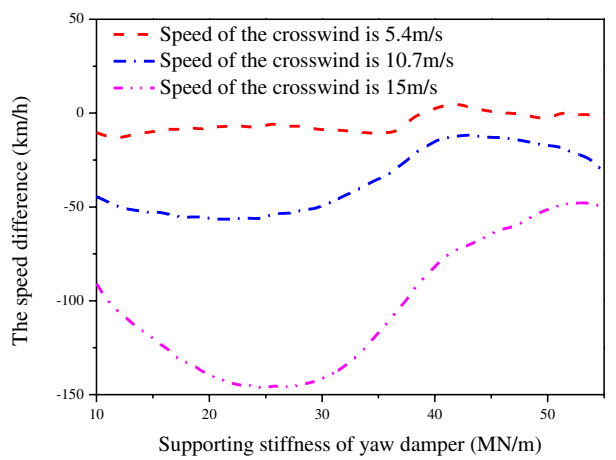

(b)

Fig. 16. (a) Linear critical speed versus supporting stiffness of yaw damper and (b) speed differences versus supporting stiffness of yaw damper.

supporting stiffness is greater than a certain value, e.g. $3 \mathrm{MN} / \mathrm{m}$. This means that for large supporting stiffness of the lateral damper, the interaction between the aerodynamic load and stiffness is small. However, the interaction is significant at small supporting stiffness. For supporting stiffness of the yaw damper, as shown in Fig. 16(b), the interaction between the aerodynamic load and stiffness is significant in the entire range. The interaction between the aerodynamic load and supporting stiffness of the lateral and yaw dampers becomes more visible as the velocity of crosswind increases.

\section{Conclusions}

The hunting stability of high-speed railway vehicles traveling on straight tracks, considering the steady aerodynamic load owing to the crosswind and airflow in the 
opposite direction of the train, was investigated by building a mathematical model for a vehicle with $17 \mathrm{DOF}$. The model takes into account the variations of wheel-rail normal forces, creep coefficients, and gravitational stiffness and angular stiffness owing to the aerodynamic load. All the above collectively contribute to the change of the linear hunting stability. The eigenvalue problem of the high-speed railway vehicle was also investigated quantitatively. The eigenvalues, eigenvectors, root locus diagram, linear critical hunting speed, and the relations among these parameters and crosswind velocity or primary and secondary suspension parameters were obtained.

The linear critical speed decreases monotonously with the increasing velocity of crosswind, and the pitching moment and lift force affect the linear critical speed more than the other components of the aerodynamic load.

Aerodynamic lift force, drag force, lateral force, and overturning moment affect the moduli of the displacement elements of the instability mode, and have little effect on the arguments. The pitching moment significantly affects the moduli and arguments of the displacement in the instability mode.

The natural period of the hunting motion visibly increases with the increasing velocity of crosswind. The attitudes of the wheelset and bogie are notably affected by the aerodynamic load. The aerodynamic load apparently changes the moduli and arguments of the elements of the eigenvectors and the phase differences between the elements. These effects become more distinct with the increasing crosswind velocity. The changes in the moduli of the lateral displacement of the wheelsets and bogie frames are more obvious, whereas the changes in the moduli of the displacement of the car body are small. The changes in the arguments of the displacement elements of hunting mode are all noticeable when the aerodynamic load is considered.

The aerodynamic load affects the linear critical speed for any primary and secondary suspension parameters even though the aerodynamic load does not affect the variation trend of the curves of speed with respect to the primary/secondary suspension parameters. Aerodynamic load and primary or secondary suspension parameters can affect the linear critical speed independently; moreover, their combined action affects the linear critical speed within a certain range of the suspension parameters.

\section{Acknowledgments}

This study was funded by the National Natural Science Foundation of China (11672306, 51490673), the Strategic Priority Research Program of the Chinese Academy of Sciences (XDB22020101), the National Key Research and Development Program of China (Grant No. 2016YFB1200602-09), the Informatization Program of the Chinese Academy of Sciences (XXH13506-204) and the National Basic Research Program (973 Program) of China (2014CB046801). 


\section{Appendix A. Vehicle Parameters}

\begin{tabular}{|c|c|}
\hline Parameters & Value \\
\hline Mass of wheelset & $m_{w}=1627 \mathrm{~kg}$ \\
\hline Mass of bogie & $m_{f}=2056 \mathrm{~kg}$ \\
\hline Mass of car body & $m_{c}=33786 \mathrm{~kg}$ \\
\hline Roll moment of the inertia of the bogie & $I_{f x}=1390 \mathrm{~kg} \cdot \mathrm{m}^{2}$ \\
\hline Roll moment of the inertia of the car body & $I_{c x}=109500 \mathrm{~kg} \cdot \mathrm{m}^{2}$ \\
\hline Pitch moment of the inertia of the car body & $I_{c y}=1555000 \mathrm{~kg} \cdot \mathrm{m}^{2}$ \\
\hline Yaw moment of the inertia of the wheelset & $I_{w}=830 \mathrm{~kg} \cdot \mathrm{m}^{2}$ \\
\hline Yaw moment of the inertia of the bogie & $I_{f z}=3800 \mathrm{~kg} \cdot \mathrm{m}^{2}$ \\
\hline Yaw moment of the inertia of the car body & $I_{c z}=1562300 \mathrm{~kg} \cdot \mathrm{m}^{2}$ \\
\hline Primary longitudinal stiffness & $K_{w x}=1.2 \times 10^{8} \mathrm{~N} / \mathrm{m}$ \\
\hline Primary lateral stiffness & $K_{w y}=1.25 \times 10^{7} \mathrm{~N} / \mathrm{m}$ \\
\hline Primary vertical stiffness & $K_{1}=886500 \mathrm{~N} / \mathrm{m}$ \\
\hline Primary vertical damping & $C_{1}=19600 \mathrm{Ns} / \mathrm{m}$ \\
\hline Half of track gauge & $b=0.7465 \mathrm{~m}$ \\
\hline Half of lateral distance of primary suspension & $b_{1}=1.02 \mathrm{~m}$ \\
\hline Half of longitudinal distance of primary suspension & $l_{t}=0.77 \mathrm{~m}$ \\
\hline Vertical distance from primary suspension to the C.G. of the bogie & $h_{f}=-0.121 \mathrm{~m}$ \\
\hline Longitudinal and lateral stiffness of the secondary suspension & $K_{2}^{\prime}=1.3 \times 10^{5} \mathrm{~N} / \mathrm{m}$ \\
\hline Vertical stiffness of the secondary suspension & $K_{2}=2.03 \times 10^{5} \mathrm{~N} / \mathrm{m}$ \\
\hline Half of the lateral distance of the secondary suspension & $b_{2}=0.95 \mathrm{~m}$ \\
\hline $\begin{array}{l}\text { Vertical distance from the C.G. of the bogie frame to the } \\
\text { secondary suspension }\end{array}$ & $h_{1}=0.398 \mathrm{~m}$ \\
\hline Vertical distance from the C.G. of the car body to the secondary suspension & $h_{2}=1.076 \mathrm{~m}$ \\
\hline Half of the longitudinal distance of the secondary suspension & $l=8.6875 \mathrm{~m}$ \\
\hline Supporting stiffness of the lateral damper & $K_{y}=4.25 \times 10^{7} \mathrm{~N} / \mathrm{m}$ \\
\hline Damping of the lateral damper & $C_{y}=1.5 \times 10^{4} \mathrm{Ns} / \mathrm{m}$ \\
\hline Vertical distance from the C.G. of the bogie frame to the lateral damper & $h_{3}=-0.105 \mathrm{~m}$ \\
\hline Vertical distance from the C.G. of the car body to the lateral damper & $h_{4}=0.966 \mathrm{~m}$ \\
\hline Supporting stiffness of the yaw damper & $K_{d}=3.5 \times 10^{7} \mathrm{~N} / \mathrm{m}$ \\
\hline Damping of the yaw damper & $C_{d}=6.72 \times 10^{5} \mathrm{Ns} / \mathrm{m}$ \\
\hline Half of the lateral distance of the yaw damper & $b_{3}=1.3225$ \\
\hline Rolling radius of the wheel & $R=0.46 \mathrm{~m}$ \\
\hline Wheel conicity & $\lambda=0.17$ \\
\hline Axle weight & $W=1.15 \times 10^{5} \mathrm{~N}$ \\
\hline Air density & $\rho=1.225 \mathrm{~kg} / \mathrm{m}^{3}$ \\
\hline Reference area & $A=9.323 \mathrm{~m}^{2}$ \\
\hline Reference length & $L=3.42 \mathrm{~m}$ \\
\hline
\end{tabular}

\section{References}

1. M. Suzuki, K. Tanemoto and T. Maeda, Aerodynamic characteristics of train/vehicles under cross winds, J. Wind Eng. Ind. Aerodyn. 91 (2003) 209-218.

2. A. Carrarini, Reliability based analysis of the crosswind stability of railway vehicles, $J$. Wind Eng. Ind. Aerodyn. 95 (2007) 493-509.

3. C. J. Baker, The simulation of unsteady aerodynamic cross wind forces on trains, J. Wind Eng. Ind. Aerodyn. 98 (2010) 88-99. 
4. M. Bocciolone, F. Cheli, R. Corradi, S. Muggiasca and G. Tomasini, Crosswind action on rail vehicles: Wind tunnel experimental analyses, J. Wind Eng. Ind. Aerodyn. 96 (2008) 584-610.

5. C. Baker and F. Cheli, Cross wind effects on road and rail vehicles, Veh. Syst. Dyn. 47 (2009) 983-1022.

6. C. Wetzel and C. Proppe, On reliability and sensitivity methods for vehicle systems under stochastic crosswind loads, Veh. Syst. Dyn. 48 (2010) 79-95.

7. D. Thomas, B. Diedrichs, M. Berg and S. Stichel, Dynamics of a high-speed rail vehicle negotiating curves at unsteady crosswind, Proc. Inst. Mech. Eng. F J. Rail Rapid Transit 224 (2010) 567-579.

8. Y. C. Cheng, C. H. Chen and C. J. Yang, Dynamics analysis of high-speed railway vehicles excited by wind loads, Int. J. Struct. Stab. Dyn. 11 (2011) 1103-1118.

9. C. Baker, H. Hemida, S. Iwnicki, G. Xie and D. Ongaro, Integration of crosswind forces into train dynamic modeling, Proc. Inst. Mech. Eng. F J. Rail Rapid Transit 225 (2011) $154-164$.

10. F. Cheli, R. Corradi and G. Tomasini, Crosswind action on rail vehicles: A methodology for the estimation of the characteristic wind curves, J. Wind Eng. Ind. Aerodyn. 104 (2012) 248-255.

11. G. Tomasini and F. Cheli, Admittance function to evaluate aerodynamic loads on vehicles: Experimental data and numerical model, J. Fluids. Struct. 38 (2013) 92-106.

12. A. H. Wickens, The dynamic stability of railway vehicle wheelsets and bogies having profiled wheels, Int. J. Solids Struct. 1 (1965) 319-341.

13. D. N. Hannebrink, H. S. H. Lee, H. Weinstock and J. K. Hedrick, Influence of axle load, track gage, and wheel profile on rail-vehicle hunting, J. Manuf. Sci. Eng. 99 (1977) $186-195$.

14. J. M. Tuten, E. H. Law and N. K. Cooperrider, Lateral stability of freight cars with axles having different wheel profiles and asymmetric loading, J. Manuf. Sci. Eng. 101 (1979) $1-16$.

15. T. Hirotsu, K. Terada, M. Hiraishi and S. Yui, Simulation of hunting of rail vehicles: The case using a compound circular wheel profile, JSME Int. J. III Vib. Control Eng. Eng. Ind. 34 (1991) 396-403.

16. C. Knudsen, E. Slivsgaard, M. Rose, H. True and R. Feldberg, Dynamics of a model of a railway wheelset, Nonlinear Dyn. 6 (1994) 215-236.

17. J. Zeng, Numerical computations of the hunting bifurcation and limit cycles for railway vehicle system, J. China Railw. Soc. 18 (1996) 13-19.

18. W. H. Zhang and Z. Y. Shen, Nonlinear stability analysis of railway vehicle system, J. China Railw Soc. 18 (1996) 29-34.

19. H. True, On the theory of nonlinear dynamics and its applications in vehicle systems dynamics, Veh. Syst. Dyn. 31 (1999) 393-421.

20. H. Yabuno, T. Okamoto and N. Aoshima, Effect of lateral linear stiffness on nonlinear characteristics of hunting motion of a railway wheelset, Meccanica 37 (2002) 555-568.

21. S. Y. Lee and Y. C. Cheng, Hunting stability analysis of high-speed railway vehicle trucks on tangent tracks, J. Sound Vib. 282 (2005) 881-898.

22. S. Y. Lee and Y. C. Cheng, Nonlinear analysis on hunting stability for high-speed railway vehicle trucks on curved tracks, J. Vib. Acoust. 127(4) (2005) 324-332.

23. Y. C. Cheng, S. Y. Lee and H. H. Chen, Modeling and nonlinear hunting stability analysis of high-speed railway vehicle moving on curved tracks, J. Sound Vib. 324 (2009) 139-160.

24. P. Kim and J. Seok, Bifurcation analysis on the hunting behavior of a dual-bogie railway vehicle using the method of multiple scales, J. Sound Vib. 329 (2010) 4017-4039. 
25. K. Zboinski and M. Dusza, Self-exciting vibrations and Hopf's bifurcation in non-linear stability analysis of rail vehicles in a curved track, Eur. J. Mech. A Solids 29 (2010) 190-203.

26. W. M. Zhai and K. Y. Wang, Lateral hunting stability of railway vehicles running on elasitc track structures, J. Comput. Nonlinear Dyn. 5 (2010) 1-9.

27. O. Polach and I. Kaiser, Comparison of methods analyzing bifurcation and hunting of complex rail vehicle models, J. Comput. Nonlinear Dyn. 7 (2012) 041005.

28. H. Dong and J. Zeng, Normal form method for large/small amplitude instability criterion with application to wheelset lateral stability, Int. J. Struct. Stab. Dyn. 14 (2014) 1350073.

29. Y. C. Cheng, C. H. Huang, C. M. Kuo and C. H. Chen, Derailment risk analysis of a tilting railway vehicle moving over irregular tracks under wind loads, Int. J. Struct. Stab. Dyn. 13 (2013) 1350038.

30. Y. B. Yang, S. V. Mac and C. H. Chen, Multi-mode coupled buffeting analysis of cablestayed bridges, Int. J. Struct. Stab. Dyn. 1 (2001) 429-453.

31. Y. C. Cheng, C. H. Chen and C. T. Hsu, Derailment and dynamic analysis of tilting railway vehicles moving over irregular tracks under environment forces, Int. J. Struct. Stab. Dyn. 17 (2017) 1750098.

32. J. J. Kalker, The computation of three-dimensional rolling contact with dry friction, Int. J. Numer. Methods Eng. 14 (1979) 1293-1307.

33. X. H. Zeng, H. Wu, J. Lai and H. Z. Sheng, Influences of aerodynamic loads on hunting stability of high-speed railway vehicles and parameter studies, Acta Mech. Sin. 30 (2014) 889-900. 